\title{
Ectopic hepatocyte transplantation cures the pig model of tyrosinemia
}

Clara T Nicolas ${ }^{1,2,3}$, Raymond D Hickey ${ }^{1,4}$, Kari L Allen ${ }^{1}$, Zeji Du ${ }^{1}$, Caitlin J VanLith, ${ }^{1}$ Rebekah M Guthman ${ }^{1,5}$, Bruce Amiot ${ }^{1}$, Lukkana Suksanpaisan ${ }^{7}$, Bing Han ${ }^{8}$, Maria Giovanna Francipane ${ }^{8,9}$, Amin Cheikhi ${ }^{10}$, Huailei Jiang ${ }^{11}$, Aditya Bansal ${ }^{11}$, Mukesh K Pandey ${ }^{11}$, Ishan Garg ${ }^{11}$, Val Lowe ${ }^{11}$, Aditya Bhagwate ${ }^{12}$, Daniel O'Brien ${ }^{12}$, Jean-Pierre A Kocher ${ }^{12}$, Timothy R DeGrado ${ }^{11}$, Scott L Nyberg $^{1}$, Robert A Kaiser ${ }^{1,6}$, Eric Lagasse ${ }^{8, *}$, Joseph B Lillegard ${ }^{1,6,13, *}$

${ }^{1}$ Department of Surgery, Mayo Clinic, Rochester, MN.

${ }^{2}$ Faculty of Medicine, University of Barcelona, Barcelona, Spain.

${ }^{3}$ Department of Surgery, University of Alabama Birmingham, Birmingham, AL.

${ }^{4}$ Department of Molecular Medicine, Mayo Clinic, Rochester, MN.

${ }^{5}$ Medical College of Wisconsin, Wausau, WI.

${ }^{6}$ Children's Hospitals and Clinics of Minnesota, Midwest Fetal Care Center, Minneapolis, MN.

${ }^{7}$ Imanis Life Sciences, Rochester, MN.

${ }^{8}$ McGowan Institute for Regenerative Medicine and Department of Pathology, University of Pittsburgh, Pittsburgh, PA.

${ }^{9}$ Ri.MED Foundation, Palermo, Italy.

${ }^{10}$ Department of Physical Medicine and Rehabilitation, University of Pittsburgh, Pittsburgh, PA.

${ }^{11}$ Department of Radiology, Mayo Clinic, Rochester, MN.

${ }^{12}$ Department of Biomedical Statistics and Informatics, Mayo Clinic, Rochester, MN.

${ }^{13}$ Pediatric Surgical Associates, Minneapolis, MN.

* These authors contributed equally to the work

To whom correspondence should be addressed:

Joseph Lillegard, M.D., Ph.D.

jlillegard@msn.com

$2001^{\text {st }}$ St SW, Rochester, MN 55902

775-224-2790

\section{One Sentence Summary:}

Transplantation of corrected hepatocytes in mesenteric lymph nodes can cure fatal metabolic liver disease by providing organized liver tissue and by repopulating the diseased liver in the pig tyrosinemia model. 


\begin{abstract}
The effectiveness of cell-based therapies to treat liver failure is limited by the diseased liver environment. Herein we provide preclinical proof-of-concept for the treatment of liver failure through hepatocyte transplantation into lymph nodes in a large-animal model of hereditary tyrosinemia type 1 (HT1), a metabolic liver disease caused by deficiency of fumarylacetoacetate hydrolase (FAH) enzyme. FAH-deficient pigs received autologous hepatocyte transplantation into mesenteric lymph nodes after ex vivo transduction with a lentiviral vector carrying the pig Fah gene. Hepatocytes showed early (6 hour) and durable ( 8 month) engraftment in lymph nodes, with reproduction of vascular and hepatic microarchitecture. Subsequently, hepatocytes migrated to and repopulated the native diseased liver. The corrected cells generated enough liver mass to clinically ameliorate disease as early as 97 days post-transplantation, with complete normalization of tyrosine levels and liver function tests. Integration site analysis defined the corrected hepatocytes in the liver as a subpopulation of hepatocytes in the lymph nodes, indicating that the lymph nodes served as a source for healthy hepatocytes to repopulate a diseased liver. Ectopic transplantation of hepatocytes cures the pig model of HT1 and presents a promising approach to the treatment of liver disease in patients with pre-existing liver damage and fibrosis.
\end{abstract}




\section{Introduction}

Nearly 14,000 patients wait annually for liver transplantation in the U.S. alone. The problem is considerably worse world-wide and represents one of the most challenging hurdles in medicine.(1) With a universal shortage of organs and limited resources, alternatives to whole organ transplantation are required to address this pandemic. Bioartificial liver devices and repopulation of decellularized liver scaffolds to create bioengineered organs for transplantation have yet to prove effective for the treatment of patients with liver failure. Cell therapy using primary hepatocytes has shown effectiveness in animal models, but the success of this approach has been very limited in the clinical setting.(2) One of the main reasons for this limited success is the inflammation, fibrosis, and scar tissue obstructing blood flow in the failing liver, which constitutes an adverse environment for hepatocyte engraftment and growth.(3)

Hereditary tyrosinemia type 1 (HT1) is an ideal disease model to study treatment options for acute and chronic liver failure in the preclinical environment. HT1 is an inborn error of metabolism of the liver caused by a deficiency of the fumarylacetoacetate hydrolase (FAH) enzyme, which is responsible for the last step of tyrosine catabolism and results in the inability to completely metabolize tyrosine.(4) Untreated, HT1 often leads to fulminant liver failure as early as a few months of life.(5) In the chronic form of the disease, FAH deficiency leads to persistent accumulation of toxic metabolites in the liver, causing oxidative damage and subsequent inflammation, fibrosis, cirrhosis along with high rates of hepatocellular carcinoma (HCC).(6, 7) In HT1, inflammatory changes and liver injury are seen within days to weeks without therapy. Pharmacologic treatment of HT1 exists in the form of 2-(2-nitro-4-trifluoromethylbenzoyl)-1,3cyclohexanedione (NTBC), a drug which inhibits tyrosine metabolism upstream of FAH, leading to the build-up of less toxic metabolites.(8)

We have previously created and characterized the porcine model of HT1 and showed that this animal is an excellent model of acute and chronic liver failure by reproducing the 
inflammation, fibrosis and cirrhosis pattern seen in many human liver diseases( 9 ). We have since demonstrated that ex vivo gene therapy involving lentiviral transfer of a functional Fah cDNA into autologous hepatocytes is curative in both mouse and pig models of HT1.(10) In our previous work, primary hepatocytes were isolated from a partial hepatectomy and transduced ex vivo by a lentiviral vector carrying a functional human Fah gene. Once corrected, cells were transplanted back into the donor animal via portal vein infusion. However, a substantial clinical limitation to our previous approach is that orthotopic hepatocyte transplantation may not be feasible in patients with acute or chronic liver disease, as the diseased liver is often an inadequate and hostile environment for hepatocyte engraftment and expansion.(11)

Alternative anatomical sites for transplantation of corrected cells could provide a healthier milieu to enable hepatocyte engraftment and proliferation. Lymph nodes are one such alternative site due to several defining characteristics. For example, they are prepared to harbor rapid expansion of $\mathrm{T}$ and $\mathrm{B}$ cells to support a swift immune response when needed.(12, 13) Lymph nodes are not only capable of accommodating immune cells, but are also a common metastatic site for many types of cancer.(14) They naturally provide a favorable environment for metastatic cell engraftment and growth. This is due, in part, to their high vascularization potential, which permits neoangiogenesis, $(15,16)$ as well as to their reticular network of fibroblasts and other stromal cells that provide physical and trophic support. $(17,18)$ Inherent plasticity, together with the fact that their systemic function is not hampered by the transplanted cells,(19) makes lymph nodes a promising site for ectopic cell delivery. Finally, lymph nodes are also easily accessible for both delivery and monitoring of the transplanted cells.

We reported that, after intraperitoneal transplantation of hepatocytes in mice, these cells colonize the lymph nodes and are able to rescue animals from lethal hepatic failure.(20) We explored the mouse lymph node as an ectopic transplantation site for multiple tissues, including liver, and demonstrated that injection of hepatocytes into a single lymph node generated enough 
ectopic liver mass to rescue the metabolic disorder in the mouse model of HT1.(21) However, it is unknown whether these promising results are translatable into a larger animal model, where a substantially higher hepatic mass would be required for rescue of liver disease. In this preclinical proof of concept study, we demonstrate the therapeutic potential of ectopic transplantation of ex vivo corrected hepatocytes into lymph nodes in the HT1 pig, a genetic model of liver failure. In tracking the fate of these cells we show the ability of ectopically transplanted hepatocytes to engraft long term in the mesenteric lymph nodes where they recreate important liver architecture that gives rise to multiple cell lineages seen in the native liver, and serve as a reservoir for repopulation of the recovering native liver.

\section{Results}

\section{Hepatocytes engraft in mesenteric lymph nodes after ectopic transplantation}

To demonstrate that hepatocytes are able to engraft in lymph nodes in a large animal model, a wildtype pig underwent a partial hepatectomy, and harvested hepatocytes were labeled with ${ }^{89} \mathrm{Zr}$ (halflife $78.4 \mathrm{~h})(22)$ prior to transplantation into 10-20 mesenteric lymph nodes. Radiolabeling efficiency was $\sim 20 \%$ and radioactivity concentration was $\sim 0.1 \mathrm{MBq} / 10^{6}$ cells. The animal received $6 \times 10^{8}$ hepatocytes through direct mesenteric lymph node injection. PET-CT imaging at 6 h posttransplantation demonstrated the presence of radioactivity within mesenteric lymph nodes $(261.8 \pm$ 108.7 SUV; Fig 1D, Video S1). Radioactivity remained present within the mesenteric lymph nodes at the 54 and $150 \mathrm{~h}$ time points $(101.1 \pm 34.7$ and $70.0 \pm 26.4 \mathrm{SUV}$, respectively). Background activity was measured in the left lumbar paraspinal muscle (0.1 SUV). Interestingly, although no radioactivity was detected within the liver at the $6 \mathrm{~h}$ time point, increasing amounts of radiotracer were found in the liver at the 54 and $150 \mathrm{~h}$ time points $(3.3 \pm 0.4$ and $5.9 \pm 0.8 \mathrm{SUV}$, respectively), indicating accumulation of ${ }^{89} \mathrm{Zr}$-labeled cells or cellular debris. The absence of radioactivity in bone, a known site of uptake for unchelated ${ }^{89} \mathrm{Zr}$, suggested that the radiolabel was retained within 
the dinitrobenzamide DBN chelator construct and not indicative of unincorporated "free" label. These data at 0.69 and 1.9 half-lives, respectively, suggest possible migration of the transplanted hepatocytes from the lymph nodes to the liver or labeled cellular debris passing through the liver. No radioactivity above background was found in spleen, lung or other organ systems at either of these time points.

\section{Ex vivo corrected hepatocytes are able to cure a pig model of HT1 after ectopic} transplantation into lymph nodes A total of $5 \mathrm{Fah}^{-/-}$animals were maintained on NTBC until the time of ex vivo-corrected autologous hepatocyte transplantation into lymph nodes (Fig 1A-C), at which point administration of NTBC was discontinued to propagate liver injury and stimulate hepatocyte regeneration. This induced selective expansion of the newly transplanted FAH-positive hepatocytes. Animals were cycled on and off NTBC based on weight parameters until NTBCindependent growth was achieved (Fig 2A, Fig S1). NTBC-independent growth was attained at a mean of $135 \pm 25$ days post-transplantation (range: 97 to 161 days), after 3 to 6 cycles of NTBC.

Biochemical cure of the HT1 phenotype was confirmed by normalization of liver specific enzymes known to be elevated in HT1 as well as normalization of tyrosine levels. At the time of euthanasia, mean tyrosine levels $(84.2 \pm 34.5 \mu \mathrm{M})$ in the five treated pigs were within normal limits for wildtype animals, and were significantly lower than untreated $\mathrm{Fah}^{-/-}$controls $(826.3 \pm 277.5 \mu \mathrm{M})($ Fig 2B). Similar results were seen in liver function tests in treated animals (AST: $56.8 \pm 21.7 \mathrm{U} / \mathrm{L}$; ALP: $186.6 \pm 78.8 \mathrm{IU} / \mathrm{L}$; ammonia: $35.8 \pm 14.4 \mu \mathrm{mol} / \mathrm{L}$; albumin: $3.6 \pm 0.3 \mathrm{~g} / \mathrm{dL}$; and total bilirubin: $0.15 \pm 0.1 \mathrm{mg} / \mathrm{dL}$ ) compared to untreated $\mathrm{Fah}^{-/}$controls $(\mathrm{AST}: 343.7 \pm 54.5 \mathrm{U} / \mathrm{L}$; ALP: $918.3 \pm$ 282.5 IU/L; ammonia: $553 \pm 561.3 \mu \mathrm{mol} / \mathrm{L}$; albumin: $2.8 \pm 0.9 \mathrm{~g} / \mathrm{dl}$; total bilirubin: $1 \pm 0.7 \mathrm{mg} / \mathrm{dl})$.

Hepatocytes transplanted into lymph nodes demonstrate long-term survival To confirm that transplanted hepatocytes were still present in mesenteric lymph nodes at later time points, two of the animals were co-transduced with a lentiviral vector carrying the Fah transgene and a second 
lentiviral vector carrying the Nis reporter gene. We performed PET/CT imaging of these two treated pigs to monitor for the expansion of NIS-positive hepatocytes in the mesentery and other tissues. One hour prior to imaging, animals received $10 \mathrm{mCi}(370 \mathrm{MBq})$ of $\left[{ }^{18} \mathrm{~F}\right] \mathrm{TFB}$. Pigs 265 and 268 were scanned at 104 and 203 days and 177 and 203 days respectively post-transplantation. Both animals showed NIS-positivity in mesenteric lymph nodes (15.7 and 39.0 SUV and 92.6 and 32.0 SUV, respectively; Fig 3A, Videos S2-5). Despite greater than 50\% repopulation of the liver at necropsy with FAH-positive hepatocytes, minimal NIS activity was seen in the liver.

To correlate NIS-positivity in mesenteric lymph nodes with histological findings, previously targeted lymph nodes were biopsied in two animals at day 140 post-transplantation. All lymph nodes samples were taken from the root of the mesentery and were grossly positive for hepatic tissue and confirmed by IHC staining for FAH expression, which would be unique to ex vivocorrected hepatocytes due to the use of the $F A H^{-/}$pig and transgenic expression controlled by the liver-specific TBG promoter (Fig 3B). Transplanted hepatocytes were more commonly found in a subcapsular location within the lymph node. All experimental animals were euthanized at 212 to 241 days post-transplantation. Histology of the collected mesenteric lymph nodes showed sustained presence of FAH-positive hepatocytes (Fig 3B, right panels), which was observed in four out of five animals. The two animals that had been biopsied previously still had FAH-positive hepatocyte engraftment in lymph nodes at necropsy confirmed by FAH and cytokeratin 18 IHC staining (Fig 3B, left and middle panels). CD31-positive endothelial cells were found within areas of hepatocyte engraftment in positive tissues (Fig 3B, Pig 268 bottom right panel), suggesting that significant blood vessel remodeling took place after transplantation to sustain long-term engraftment and hepatic growth. Positivity for glutamine synthetase, an enzyme exclusively expressed in pericentral hepatocytes in mammals,(23) was found in hepatocytes surrounding CD31 positive neo-vessels, suggesting neo-formation of a central vein and, importantly, proper physiological zoning of the ectopic liver. Reproduction of hepatic microarchitecture within transplanted lymph nodes was 
further supported by the presence of hepatic lobules similar to those observed in native pig livers, and cytokeratin 7-positive bile duct cells within the areas of engraftment (Fig 3B, Pig 265 bottom right panel).

Hepatocytes transplanted into lymph nodes migrate to the liver Migration of transplanted hepatocytes to the liver was confirmed by FAH IHC of liver tissue demonstrating multiple FAHpositive nodules within the livers of all five animals, covering 67 to $100 \%(84.7 \pm 7.0)$ of the total liver area at the time of necropsy. A negative correlation was found between percent liver repopulation with FAH-positive hepatocytes and sustained presence of FAH-positive areas of hepatocyte engraftment within lymph nodes; one pig with complete liver repopulation with FAHpositive hepatocytes showed little to no hepatocyte presence remaining within the lymph nodes at the terminal time point, while pigs with only partial liver repopulation with FAH-positive hepatocytes demonstrated a more robust hepatocyte presence within the lymph nodes at 235-239 days post-transplantation (Fig S2). As expected, FAH-negative areas of the liver showed marked hepatocellular damage and fibrosis due to prolonged NTBC withdrawal. However, the two fully FAH-positive livers demonstrated healthy, normal-looking tissue with minimal residual fibrosis, suggesting that the hepatic insult that occurs during NTBC cycling is reversible with time, as FAHpositive hepatocytes expand to repopulate the liver (Fig 4).

In order to characterize any differences between the hepatocytes present in the liver and those present in the lymph nodes, we performed next generation sequencing and bioinformatics analysis of both cell populations. Mapping statistics are provided in Table S1. We found no significant differences in general lentiviral integration profile between these two cell populations. In both cases, integration occurred more often in coding regions than in non-coding regions of the genome, with exons being preferred over introns (Fig 5A). Therefore, integration was favored in chromosomes with higher gene densities (Fig S3B). In both cell populations, lentiviral integration was rare in CpG-rich islands and was clearly favored downstream of transcription start sites (Fig 
5B and $\mathrm{C}$ ), suggesting minimal tumorigenicity potential from oncogene activation. Furthermore, there was no preference for integration in tumor-associated genes in either cell population (Fig 5D).

The genes with the highest integration frequency in both groups of cells are presented in Table 2, and it is here that differences between the two cell populations were found. Fah was the gene with the highest number of distinct integration sites in both groups, although it was only in the top 10 integrated genes in the lymph node population. Interestingly, all but two of the 135 unique integration sites within Fah locus in the liver hepatocyte population were represented in the 206 integration sites present in the lymph node hepatocyte population (Fig 5E), indicating that the transplanted cells first engrafted in the lymph nodes, divided and then, after clonal expansion, migrated to the liver. The top four genes with the highest integration frequency in the liver population were also within the top 20 genes in the lymph node population, and were all approximately 5-fold enriched in the liver population when evaluated by the total number of reads present for each, suggesting unbiased expansion and enrichment of a subpopulation of lymph node hepatocytes within the liver. These genes were: Mir9799, microRNA; Ndufv2, NADH dehydrogenase ubiquinone flavoprotein 2; Ifrd1, interferon-related developmental regulator 1; and Suclg1, succinyl-CoA ligase (GDP-forming), alpha subunit. In native and ectopic liver samples obtained from our experimental animals after necropsy, expression of Suclg1, Ndufv2, and Ifrd1 was indeed disrupted compared to control liver levels, possibly due to the insertion of the LV construct in at least one allele in some of the corrected hepatocytes (Fig 5F). As a control, NIS expression was evaluated and only detectable in 2 of the 3 samples of hepatized lymph nodes analyzed. The fifth most prevalent integration site in the hepatocytes that migrated to the liver was Cenpp, centromere protein $\mathrm{P}$, which was enriched over 78-fold compared to the lymph node population and could be an indication that this disruption caused a favorable migration, engraftment, and/or expansion profile for these clones specifically. 
To determine whether hepatocytes transplanted in lymph nodes generated a tissue resembling normal liver, transcriptome profiles of untransplanted and hepatized lymph nodes, as well as native (engrafted) and control (isolated from a healthy, untransplanted pig) livers were compared. Twelve thousand genes were identified as differentially expressed (DE) and contrasting control lymph node to liver tissues including hepatized lymph nodes (Figure 6A). T-distributed Stochastic Neighbor Embedding (t-SNE) showed a strong tendency of hepatized lymph node transcripts to cluster with liver tissues samples, whereas control lymph nodes were distinct from the other three groups (Figure 6B). These data suggest that a significant proportion of the lymph node transcriptome acquires a liver-like signature after hepatocyte transplantation. To estimate the similarity of hepatized lymph nodes with control liver, we first focused on 24 genes, which were previously described to be liver-specific (LiGEP, Table S2 and Figure S4). We then included 20 additional genes in our analysis, which can be classified as cytochrome P450 genes, other enzymes, plasma proteins, transporters, surface molecules and cytokines, and transcription factors (Table S2). Cluster heat map showing the RNAseq expression levels of all 44 genes is shown in Figure 6C. Evaluation of albumin expression demonstrates adoption of a liver phenotype by hepatized lymph nodes relative to control (Fig 6D, left) while analysis of $F A H$ shows transgenic expression in hepatized lymph nodes as well as elevated transgenic expression in repopulated native liver (under TBG promoter) relative to control liver (Fig 6D, right). Expression levels of all other genes are shown in Fig S4A (LiGEP) and S4B (additional liver-specific genes).

\section{Discussion}

In many human liver diseases, orthotopic hepatocyte transplantation and engraftment is often limited by the presence of significant liver injury in the form of inflammation, fibrosis and cirrhosis,(11) underscoring the importance of evaluating alternative transplantation sites. Ectopic hepatocyte transplantation has been attempted in several preclinical and clinical studies.(19) Of the alternative sites tested, only spleen and peritoneal cavity have been able to accommodate a 
clinically significant number of cells.(24) Intrasplenic injection of hepatocytes improves liver function and prolongs survival in a mouse model of liver failure.(25) Although transsplenic access to the portal vein in human patients has been done (26), this may introduce hemorrhagic risk that is decreased with lymph node transplantation. Intraperitoneal transplantation of hepatocytes has been attempted in humans with acute liver failure, but transplanted cells are not able to survive in this environment for extended periods of time.(27)

Lymph nodes are a highly favorable site for ectopic cell transplantation.(19) Not only does their plasticity and highly complex vascular network provide a healthy milieu for cell engraftment and expansion, as demonstrated by both their natural interactions with lymphocytes and their pathological interactions with metastatic tumor cells, but their accessibility makes it possible to perform minimally invasive cell delivery in the clinical setting. This could prove especially important in patients that are higher risk surgical candidates. There is ample precedent in the clinic for ultrasound-guided lymph node identification and targeting. Fine needle aspiration biopsies, for example, are now a routine diagnostic test. Multiple clinical studies have also made use of lymph nodes as sites for injection of immunotherapeutic agents, with patients rating the procedure comparable to a subcutaneous injection and less painful than venous puncture.(28) Furthermore, cell transplantation into lymph nodes does not appear to affect their function: no complications were noted in our study or in previous studies.(20) (21)

All five animals treated through this method demonstrated NTBC independence within three to five months of treatment, as well as complete normalization of tyrosine levels and liver function tests. This timeframe to metabolic correction is comparable to that of our previous study using similar numbers of cells and portal vein delivery into the native FAH-deficient liver.(10) It is worth noting that in our previous study, animals were also maintained on the protective drug NTBC until the time of transplantation, which means that their livers did not have a significant amount of inflammation and fibrosis when they received the corrected hepatocytes. Orthotopic cell 
transplantation has shown low rates of engraftment, especially when the liver is compromised by inflammation and fibrosis, as is seen in many liver diseases.(29)

It has been previously shown that hepatocyte transplantation into lymph nodes is able to generate enough ectopic liver mass to correct HT1 and rescue mice from acute and chronic liver disease.(21) In our study, we show a similar result in the large animal model of HT1, suggesting that hepatocyte transplantation into lymph nodes is scalable and may therefore be a clinically relevant technique that permits the creation of sufficient ectopic liver mass to significantly impact liver function. There are important differences between these two preclinical studies. Syngeneic and allogeneic hepatocytes were used in the HT1 mouse study by Komori et al, and the HT1 mouse does not exhibit the full extent of liver injury seen in humans, including fibrosis and cirrhosis.(21) The current study was conducted in the HT1 pigs initially supported on NTBC, where liver disease could be delayed until the harvesting of autologous cells was complete. In this study we take advantage of the porcine HT-1 model of liver failure because 1-2 weeks after the withdrawal of NTBC we begin to see rapid and severe liver injury that includes all the same features we see in human liver failure including, inflammation, fibrosis and cirrhosis. This study design allows us to isolate the single variable related to ectopic transplantation of hepatocytes in LNs in the setting of liver failure while avoiding potential confounding variables related to immune suppression or the use of primary hepatocyte alternatives. Now that we have demonstrated the feasibility of targeting LNs for hepatocyte transplantation, subsequent evaluations should be performed in untreated HT1 pigs or other large animal models of liver disease using primary hepatocyte alternatives or allotransplantation and immune suppression.

The fact that our HT1 model, which develops all the acute and chronic liver failure features seen in many other liver diseases, was completely weaned of NTBC requirement and metabolically normalized after the ectopic transplantation procedure indicates the therapeutic potential of this approach. Even though the goal of this study was not to evaluate directly the use of ectopic LN 
hepatocyte transplantation for the treatment of any specific genetic liver disease, there are some potential applications that should be discussed regarding these diseases. Some optimizations in initial engraftment rates or other modifications (i.e., exogenously applied selective pressure or repeat administrations of corrected cells) would be necessary for diseases that do not inherently propagate healthy cells, such as phenylketonuria or Wilson's disease. However, it is the liver damage itself through the hepatectomy and not something specific to HT1 that provides the stimulus for initial successful engraftment. Additionally, unlike HT1, most liver diseases do not require complete repopulation of the diseased liver, and administration could be catered to effect to see a phenotypic change.

Autologous hepatocyte transplantation could be directly translated into human patients where their condition does not preclude a partial hepatectomy, thereby avoiding immune concerns related to the use of allogeneic cells. However, healthy allogeneic hepatocytes could be transplanted in cases where partial hepatectomy is contraindicated, such as in acute liver failure or severe cirrhosis, which would require additional support via established immune suppression protocols. Possible future sources of hepatocytes include universal donor hepatocytes, IPS-derived hepatocytes, and HLA-matched farmed hepatocytes (30-32). In our study hepatocytes were transplanted into pig mesenteric lymph nodes using an open technique due to the paucity of suitable peripheral lymph nodes in the neonatal pig, but the same procedure could foreseeably be performed into central or peripheral lymph nodes in humans via a percutaneous or endoscopic ultrasound-guided technique, as described previously.(28)

In our model, we found that although hepatocytes demonstrated long-term survival within lymph nodes, forming appropriate liver architecture including bile ducts, some also subsequently engrafted in the liver after a period of engraftment and expansion in the LNs. This was suggested by bioinformatics data and confirmed by histology. This phenomenon was very limited in previous mouse studies(21) and could be related to the microanatomy and direction of flow in pig lymph 
nodes, which differ from those of other mammals. Their microarchitecture is inverted, with the germinal centers being located internal to the medulla, which has led authors to suggest that lymphocytes are transported back into a capillary system after passing through a lymph node, as opposed to the lymphatic system as in other animals. $(33,34)$ Indeed, during the injection procedure it was possible to observe injected fluid communicating from the lymph node into the capillary bed.

The fate of the engrafted hepatocytes into the lymph nodes was assessed by the evaluation of lentiviral vector integration into prevalent site, such as the Fah locus. Of 135 unique integration sites in the Fah locus seen in the hepatocytes engrafted in the liver, only 1 was not among the 206 unique integration sites observed in the hepatocytes in lymph nodes. This result substantiates the conclusion that although cells migrate from the lymph nodes to the liver after transplantation they do so primarily after a period of engraftment in the lymph nodes. If the hepatocytes had migrated immediately to the liver after transplantation we would have expected a more disparate integration profile between the two populations. Further experiments conducted on a different large animal model with a similar lymphatic anatomy to humans would be required to judge whether the level of hepatocyte migration from mesenteric lymph nodes to liver remains the same. If so, central lymph node injection could potentially be considered a safer alternative to portal vein injection for hepatocyte delivery into the liver, since portal vein infusion of cells often leads to thrombotic complications and requires systemic anticoagulation.(35)

Based on these results, we also analyzed in detail the overall integration profile of our lentiviral vector in both hepatocytes that remained in the lymph nodes and hepatocytes that migrated to the liver. In both cell populations, the lentiviral vector showed a benign integration profile, with no preference for integration in promoter regions or tumor-associated genes. Analysis of the genes with the highest integration frequency in both populations suggested that hepatocytes that migrated to and expanded in the liver were a subpopulation of hepatocytes that remained in the lymph nodes, although discerning any functional impact of disrupting the integrated genes was beyond the scope 
of the current study. Interestingly, prevalence of the top four integrated genes in the liver population was approximately five-fold higher than in the lymph node population, a linearity possibly supported by a clonal founder with all four integrations or up to 4 unique clonal founders with identical (unbiased) expansion after migration and liver engraftment. Of note, Fah was within the top twenty genes with the highest integration frequency and was the gene with the highest number of distinct integration sites in both groups. This suggests that homology may guide integration to some extent, a finding that provokes further development to possibly improve safety and predictability of lentiviral genomic disruption.

We also examined relative changes in gene expression, finding no significant differences in general transcriptomes between engrafted and control lymph nodes, as well as engrafted and control liver, aside from specific variations induced by transgene expression (i.e. $F A H$ ) or lymph node adoption of a liver phenotype (i.e., albumin expression). This implies that transplantation of hepatocytes in lymph nodes and ex vivo lentiviral gene therapy does not significantly alter endogenous gene expression within the corrected cells. Together with the integration profiling data (discussed above), this advocates for the safety of the development of an ectopic liver in lymph nodes and the ex vivo lentiviral gene therapy approach. Given the clinical success of ex vivo lentiviral gene therapy in cancer via CAR-T cells (see review by Geyer and Brentjens, 2016) and the over 250 open FDA trials using lentivirus, our data support progression of this paradigm into additional indications. Ultimately, approval of expanded clinical application of ex vivo lentiviral gene therapy will be made on a risk:reward basis, and a growing body of literature from our laboratories and others support both safety (reduced risk) and efficacy (increased reward) of this approach.

In summary, we have shown that ectopic transplantation of corrected, autologous hepatocytes into mesenteric lymph nodes is curative in the HT1 pig model of severe liver failure. Cells transplanted into these ectopic sites engraft and expand creating hepatized LNs, aiding the failing liver. Hepatocyte transplantation into lymph nodes is a promising approach to the treatment of multiple 
liver diseases that holds several important advantages over more traditional cell transplantation techniques, especially in patients with pre-existing native liver damage.

\section{Materials and Methods}

Study design This prospective controlled laboratory experiment was designed to address the hypothesis that ectopic transplantation of hepatocytes would result in sufficient functional biomass to support a large animal model of liver failure, specifically the porcine model of hereditary tyrosinemia type I (HTI) during NTBC withdrawal. Five HT1 pigs underwent ex vivo gene therapy with two lentiviral vectors (one expressing FAH and the other expressing the NIS reporter gene). The NIS reporter should not affect phenotype but is only intended to allow non-invasive tracking of transplanted cells. The finding that ectopic transplantation of corrected hepatocytes seeded subsequent robust orthotopic engraftment was not anticipated, and the additional hypothesis that the hepatocytes that migrated to liver were a subset of those that were initially engrafted in the lymph nodes (as opposed to a unique population that never engrafted ectopically) was further developed and tested using bioinformatics.

These studies in large animals were performed in a limited number of subjects $(n=5)$ due to resource-intensive nature of the involved surgical and diagnostic procedures in large animals, and associated resource requirements for the husbandry, in vivo evaluations, and bioinformatic analyses.

Animals All animal procedures were performed in compliance with Mayo Clinic's Institutional Animal Care and Use Committee regulations and all animals received humane care. For biodistribution experiments, a female wildtype pig was used. For ex vivo gene therapy experiments, male and female $\mathrm{Fah}^{-/-}$pigs were used. $\mathrm{Fah}^{-/-}$pigs were produced in a 50\% Large White and 50\% Landrace pig as previously described. $(36,37)$ 
NTBC administration NTBC mixed in food was administered at a dose of $1 \mathrm{mg} / \mathrm{kg} / \mathrm{day}$ with a maximum of $25 \mathrm{mg} /$ day. All animals remained on NTBC until the time of transplantation, after which NTBC administration was discontinued to support expansion of the corrected cells. After hepatocyte transplantation, all animals were monitored daily for loss of appetite or any other clinical signs of morbidity. Animals were weighed daily for the first two weeks post-operatively and weekly thereafter. If loss of appetite, weight loss, or any other signs of morbidity occurred, NTBC treatment was reinitiated for seven days. Animals were cycled on and off NTBC in this fashion to stimulate expansion of corrected FAH-positive cells.

Liver resection and hepatocyte isolation Eight-week-old (16-21 kg) pigs underwent a laparoscopic partial hepatectomy involving the left lateral lobe under inhaled general anesthesia with $1-3 \%$ isoflurane. Resection volumes represented 15 to $20 \%$ of the total liver mass. Using an open Hasson Technique, a $12 \mathrm{~mm}$ port was placed into the peritoneal cavity and the abdomen insufflated. Using a $5 \mathrm{~mm}$ laparoscope (Stryker, Kalamazoo, MI), two additional $5 \mathrm{~mm}$ ports were placed under direct visualization. The left lateral segment and its vascular and biliary drainage was isolated and divided using an Endo GIA stapler (Covidien, Dublin, Ireland). The liver section was retrieved using an Endo Catch bag (Covidien, Dublin, Ireland), and adequate hemostasis was ensured prior to port removal and incision closure. This liver section was then perfused ex vivo through the segmental portal vein with a two-step perfusion system to isolate hepatocytes as previously described.(38) Number and viability of cells were determined by trypan blue exclusion.

Hepatocyte radiolabeling For early biodistribution experiments, hepatocytes were radiolabeled in suspension with ${ }^{89} \mathrm{Zr}\left(\mathrm{t}_{1 / 2}=78.4 \mathrm{hr}\right)$ with synthon ${ }^{89} \mathrm{Zr}-\mathrm{DBN}$ at $27^{\circ} \mathrm{C}$ for $45 \mathrm{~min}$ in Hank's Buffered Salt Solution (HBSS) as previously described. (39-41)

Hepatocyte transduction Hepatocytes were co-transduced in suspension at a MOI of 20TUs with two third-generation lentiviral vectors carrying the sodium-iodide symporter (Nis) reporter gene or the pig Fah gene under control of the thyroxine-binding globulin promoter (Fig 1A). The NIS reporter allows for longitudinal non-invasive monitoring of transplanted cells through single- 
photon emission computed tomography (SPECT) or positron emission tomography (PET).(42) Transduction occurred over the course of $2 \mathrm{~h}$ before transplantation using medium and resuspension techniques previously described.(10) Hepatocytes were resuspended in 0.9\% sodium chloride (Baxter Healthcare Corporation, Deerfield, IL).

Hepatocyte transplantation in pigs All pigs received autologous transplantation of hepatocytes through direct mesenteric lymph node injection (Fig 1B). After partial hepatectomy, animals were kept under general anesthesia until the time of transplantation, approximately $4 \mathrm{~h}$ later.. Bowel was exteriorized through the upper midline incision until the root of the mesentery was visible (Fig 1C). Mesenteric lymph nodes were identified and hepatocytes were delivered directly into 10-20 nodes through direct injection with a 25-gauge one-inch needle. Each animal received a total of $6 \times 10^{8}$ hepatocytes in 10-20 $\mathrm{mL}$ of saline. Heparinization of the cell solution just prior to injection was performed at $70 \mathrm{U} / \mathrm{kg}$ of recipient weight as has been previously described for islet cell transplantation protocols.(43)

\section{Positron emission tomography-computed tomography (PET-CT) imaging and analysis}

Imaging was performed on the high-resolution GE Discovery 690 ADC PET/CT System (GE Healthcare, Chicago, IL) at 6, 54, and $150 \mathrm{~h}$ post-transplantation in the ${ }^{89} \mathrm{Zr}$-labeled animal. Noninvasive evaluation of NIS expression was performed by PET/CT at 3, 5, and 6 month posttransplantation in two of the NIS-labeled animals using $\left[{ }^{18} \mathrm{~F}\right]$ tetrafluoroborate $\left(\left[{ }^{18} \mathrm{~F}\right] \mathrm{TFB}\right)$ radiotracer.(44) CT was performed at $120 \mathrm{kV}$ and $150 \mathrm{~mA}$, with tube rotation of $0.5 \mathrm{~s}$ and pitch of 0.516. PET was performed as a two-bed acquisition with $10 \mathrm{~min}$ per bed and 17-slice overlap, resulting in a 27-cm axial field of view. PMOD (version 3.711; PMOD Technologies, Switzerland) was used for image processing, 3D visualization and analysis. The anatomic location of liver and kidneys was identified from the registered datasets. Volumes of interest (VOIs) of mesenchymal lymph nodes were created on PET images, and standardized uptake values (SUVs) for body weight were obtained. Surface rendering was performed using the threshold pixel value for bone, liver and 
kidneys from the CT dataset and lymph nodes from the PET dataset to localize the lymph nodes with reference to the skeleton.

Biochemical analysis Standard serum and plasma analyses were performed. Tyrosine values in plasma were determined using liquid chromatography and tandem mass spectrometry via Mayo Clinic's internal biochemical PKU test.

Histopathological and immunohistochemical analyses For H\&E, Masson's Trichrome and FAH stains, tissue samples were fixed in 10\% neutral buffered formalin (Azer Scientific, Morgantown, PA), paraffin embedded and sectioned $(5 \mu \mathrm{m})$. H\&E and Masson's Trichrome stains were performed by means of standard protocols, while immunohistochemistry for FAH was performed as previously described.(42) Percent of FAH-positive hepatocytes was quantified using a cytoplasmic stain algorithm in Aperio ImageScope. For all other immunohistochemical stains, tissues were fixed in $4 \%$ paraformaldehyde for $4 \mathrm{~h}$, infiltrated with $30 \%$ sucrose overnight and then embedded in OCT compound prior to sectioning $(5-7 \mu \mathrm{m})$. Sections were washed with phosphatebuffered saline (PBS) and blocked with 5\% bovine serum albumin (BSA) or milk for $30 \mathrm{~min}$. Sections were then incubated with primary antibody for $1 \mathrm{~h}$ and secondary antibody for $30 \mathrm{~min}$, and were coverslipped with a glycerol-based mounting media containing Hoechst. Images were captured with an Olympus IX71 inverted microscope. The following primary antibodies were used: ER-TR7 (ab51824, Abcam, Cambridge, MA), Glutamine Synthetase (ab64613, Abcam, Cambridge, MA), CD31 (MCA1746GA, Bio-Rad, Hercules, CA), Cytokeratin 7 (ab9021, Abcam, Cambridge, MA) and Cytokeratin 18 (10830-1-AP, Proteintech, Chicago, IL).

\section{Amplification, next-generation sequencing (NGS), and genomic DNA mapping of lentiviral}

integration sites Genomic DNA was isolated from snap-frozen tissue fragments of hepatized mesenteric lymph nodes and engrafted livers from Animal No. 265 using a Gentra Puregene Tissue Kit (Qiagen, Hilden, Germany). Ligation-mediated PCR (LM-PCR) was used for efficient isolation of integration sites. Restriction enzyme digestions with MseI were performed on genomic DNA samples; the digested DNA samples were then ligated to linkers and treated with ApoI to limit 
amplification of the internal vector fragment downstream of the 5' LTR. Samples were amplified by nested PCR and sequenced using the Illumina HiSeq 2500 Next-Generation Sequencing System (San Diego, CA). After PCR amplification, amplified DNA fragments included a viral, a pig and sometimes a linker segment. The presence of the viral segment was used to identify reads that report a viral mediated integration event in the genome. The reads sequenced from these DNA fragments were processed through quality control, trimming, alignment, integration analysis, and annotation steps.

Quality control of the sequenced read pairs was performed using the FASTQC software. The average base quality of the sequenced reads was $>\mathrm{Q} 30$ on the Phred scale. Uniform distribution of A,G,T and C nucleotides was seen across the length of the reads without bias for any specific bases. The number of unknown " $N$ " bases was less than $1 \%$ across the length of the reads. High sequence duplication ( $>80 \%$ ) was observed, however, this was anticipated due to the nature of the experiment and the amplification of specific library fragments.

We used Picard software's (http://broadinstitute.github.io/picard/) insert size metrics function to calculate the average fragment length per sample. This metric averaged across all the samples was 158 base pairs (bps) long with an average standard deviation of 29bps. Since 150bp long reads were sequenced, most of the paired read one (R1) and read two (R2) reads overlapped, providing redundant information. $\mathrm{R} 2$ reads were therefore not considered in the analysis.

Reads were trimmed to remove the viral sequence in two separate steps. In step 1, the viral sequence was trimmed from the $\mathrm{R} 1$ reads using cutadapt(45) with a mismatch rate $(\mathrm{e}=0.3)$ from the 5 ' end of each read. In step 2, if the linker sequence was present, it was similarly trimmed from the 3'end of the read. Trimmed reads with a length less than $15 \mathrm{bps}$ were removed from the rest of the analysis to reduce ambiguous alignments. Untrimmed reads were also removed from the rest of the analysis because they did not contain a viral segment that could be used as evidence of a viral mediated integration. 
The remaining R1 reads were aligned to the susScr11 build of the pig reference genome using BWA-MEM in single-end mode.(46) Default BWA-MEM parameters were used. The reads used to identify genomic points of viral integrations had to be uniquely mapped to the genome with a BWA-MEM mapping quality score greater than zero.

An integration point was defined by the position of R1's first aligned base on the susScr1 genome. Unique integration points were identified across the genome without any constraints on coverage. However, for downstream annotation and analysis, only those integration points with 5 or more supporting reads were used to minimize calling false positive integration points.

Locations of integration points were categorized in: exons, introns, 3 prime UTRs, 5 prime UTRs and intergenic regions using information extracted from the susScr11 refflat file maintained by UCSC's Genomics Institute. To avoid conflict of feature categorization arising from multiple overlapping gene definitions, only the definition of the longest gene was used to annotate integration points. Additional features where computed including: the distance to the nearest transcription start site (TSS), makeup of CpG-rich regions of the genome ("CpG islands"), and enrichment to tumor-associated genes. This tumor gene list was comprised of 745 clinically validated tumor-associated human genes (from Mayo Clinic internal data). Those genes were related to their pig homologs based on GenBank's gene names.

RNA isolation, tissue screening, and whole transcriptome sequencing (RNAseq) Total RNA was isolated from snap-frozen tissue fragments of hepatized or control (non-transplanted) mesenteric lymph nodes from three experimental animals, and of engrafted livers from the same three experimental animals or control liver from one untreated, control animal using the RNeasy Mini Kit (Qiagen, Valencia, CA) according to manufacturer's instructions (including the optional on-column DNase digestion). RNA purity and concentration were measured using a NanoDrop 2000/c spectrophotometer (Thermo Fisher Scientific, Pittsburgh, PA). Screening of hepatized lymph nodes was performed by examining albumin and fah expressions using quantitative reverse 
transcription PCR (qRT-PCR). Non-transplanted mesenteric lymph nodes and a control liver were used as negative and positive controls, respectively. RNA was retrotranscribed using the iScript Reverse Transcription Supermix (Bio-Rad, Hercules, CA) and albumin and fah were amplified using the SsoAdvanced ${ }^{\mathrm{TM}}$ Universal SYBR ${ }^{\circledR}$ Green Supermix (Bio-Rad, Hercules, CA) on a StepOnePlus Real-Time PCR System (Applied Biosystems, Foster City, CA). Expression of glyceraldehyde-3-phosphate dehydrogenase (GAPDH) was used for normalization of gene expression data. Relative changes in gene expression were calculated using the $2^{-\Delta \Delta C T}$ method. RNAs from selected samples were shipped on dry ice to Novogene in Sacramento, CA for library preparation and sequencing. All samples passed Novogene internal quality control with RNA integrity number above eight.

RNAseq data analysis was performed using Partek Genomics Suite software version 7.0. Briefly, quality control was measured considering sequence-read lengths and base-coverage, nucleotide contributions and base ambiguities, quality scores as emitted by the base caller, and overrepresented sequences. All the samples analyzed passed all the QC parameters and were mapped to the annotated pig reference SScrofa11.1_90+nonchromosomal using STAR2.4.1d index standard settings. Estimation of transcript abundance based on the aligned reads was performed by optimization of an expectation-maximization algorithm and expressed as FPKM (Fragments Per Kilobase Million). Differentially expressed (DE) genes were detected using differential gene expression (GSA) algorithm based on p-value of the best model for the given gene, false discovery rate (FDR), ratio of the expression values between the contrasted groups, fold change (FC) between the groups and least-square means (adjusted to statistical model) of normalized gene counts per group. 12000 genes were identified as DE based on $\log 2|\mathrm{FC}| \geq 2$ and FDR $\geq 0.05$ cut-off. Unsupervised clustering to visualize expression signature was performed using 1-Pearson correlation distance and complete linkage rule and samples were classified using t-SNE (tdistributed stochastic neighbor embedding). 
Statistical analysis Numerical data are expressed as mean \pm SD (standard deviation) or SEM (standard error of the mean). Statistical significance was determined by Welch's t-test, and established when $\mathrm{p} \leq 0.05$. Statistical analyses were performed with GraphPad Prism software version 7.

\section{References}


1. D. Shafran, E. Kodish, A. Tzakis, Organ shortage: the greatest challenge facing transplant medicine. World J Surg 38, 1650-1657 (2014).

2. K. A. Soltys, K. Setoyama, E. N. Tafaleng, A. Soto Gutierrez, J. Fong, K. Fukumitsu, T. Nishikawa, M. Nagaya, R. Sada, K. Haberman, R. Gramignoli, K. Dorko, V. Tahan, A. Dreyzin, K. Baskin, J. J. Crowley, M. A. Quader, M. Deutsch, C. Ashokkumar, B. L. Shneider, R. H. Squires, S. Ranganathan, M. Reyes-Mugica, S. F. Dobrowolski, G. Mazariegos, R. Elango, D. B. Stolz, S. C. Strom, G. Vockley, J. Roy-Chowdhury, M. Cascalho, C. Guha, R. Sindhi, J. L. Platt, I. J. Fox, Host conditioning and rejection monitoring in hepatocyte transplantation in humans. J Hepatol 66, 987-1000 (2017).

3. D. Schuppan, N. H. Afdhal, Liver cirrhosis. Lancet 371, 838-851 (2008).

4. B. Lindblad, S. Lindstedt, G. Steen, On the enzymic defects in hereditary tyrosinemia. Proc Natl Acad Sci U S A 74, 4641-4645 (1977).

5. D. C. Bartlett, C. Lloyd, P. J. McKiernan, P. N. Newsome, Early nitisinone treatment reduces the need for liver transplantation in children with tyrosinaemia type 1 and improves post-transplant renal function. J Inherit Metab Dis 37, 745-752 (2014).

6. M. Grompe, The pathophysiology and treatment of hereditary tyrosinemia type 1. Semin Liver Dis 21, 563-571 (2001).

7. F. Endo, M. S. Sun, Tyrosinaemia type I and apoptosis of hepatocytes and renal tubular cells. J Inherit Metab Dis 25, 227-234 (2002).

8. S. Lindstedt, E. Holme, E. A. Lock, O. Hjalmarson, B. Strandvik, Treatment of hereditary tyrosinaemia type I by inhibition of 4-hydroxyphenylpyruvate dioxygenase. Lancet 340, 813-817 (1992).

9. F. Elgilani, S. A. Mao, J. M. Glorioso, M. Yin, I. D. lankov, A. Singh, B. Amiot, P. Rinaldo, R. J. Marler, R. L. Ehman, M. Grompe, J. B. Lillegard, R. D. Hickey, S. L. Nyberg, Chronic Phenotype Characterization of a Large-Animal Model of Hereditary Tyrosinemia Type 1. Am J Pathol 187, 3341 (2017).

10. R. D. Hickey, S. A. Mao, J. Glorioso, F. Elgilani, B. Amiot, H. Chen, P. Rinaldo, R. Marler, H. Jiang, T. R. DeGrado, L. Suksanpaisan, M. K. O'Connor, B. L. Freeman, S. H. Ibrahim, K. W. Peng, C. O. Harding, C. S. Ho, M. Grompe, Y. Ikeda, J. B. Lillegard, S. J. Russell, S. L. Nyberg, Curative ex vivo liverdirected gene therapy in a pig model of hereditary tyrosinemia type 1. Sci Trans/ Med 8, 349ra399 (2016).

11. R. D. Hughes, R. R. Mitry, A. Dhawan, Current status of hepatocyte transplantation. Transplantation 93, 342-347 (2012).

12. U. H. von Andrian, T. R. Mempel, Homing and cellular traffic in lymph nodes. Nat Rev Immunol 3, 867-878 (2003).

13. T. Junt, E. Scandella, B. Ludewig, Form follows function: lymphoid tissue microarchitecture in antimicrobial immune defence. Nat Rev Immunol 8, 764-775 (2008).

14. J. P. Sleeman, W. Thiele, Tumor metastasis and the lymphatic vasculature. Int J Cancer 125, 27472756 (2009).

15. P. Saharinen, T. Tammela, M. J. Karkkainen, K. Alitalo, Lymphatic vasculature: development, molecular regulation and role in tumor metastasis and inflammation. Trends Immuno/ 25, 387-395 (2004).

16. T. Tammela, K. Alitalo, Lymphangiogenesis: Molecular mechanisms and future promise. Cell 140, 460-476 (2010).

17. A. Link, T. K. Vogt, S. Favre, M. R. Britschgi, H. Acha-Orbea, B. Hinz, J. G. Cyster, S. A. Luther, Fibroblastic reticular cells in lymph nodes regulate the homeostasis of naive T cells. Nat Immunol 8 , 1255-1265 (2007).

18. A. L. Fletcher, S. E. Acton, K. Knoblich, Lymph node fibroblastic reticular cells in health and disease. Nat Rev Immunol 15, 350-361 (2015).

19. A. D. DeWard, J. Komori, E. Lagasse, Ectopic transplantation sites for cell-based therapy. Curr Opin Organ Transplant 19, 169-174 (2014).

20. T. Hoppo, J. Komori, R. Manohar, D. B. Stolz, E. Lagasse, Rescue of lethal hepatic failure by hepatized lymph nodes in mice. Gastroenterology 140, 656-666 e652 (2011). 
21. J. Komori, L. Boone, A. DeWard, T. Hoppo, E. Lagasse, The mouse lymph node as an ectopic transplantation site for multiple tissues. Nat Biotechnol 30, 976-983 (2012).

22. Y. Zhang, H. Hong, W. Cai, PET tracers based on Zirconium-89. Curr Radiopharm 4, 131-139 (2011).

23. R. Gebhardt, A. Baldysiak-Figiel, V. Krugel, E. Ueberham, F. Gaunitz, Hepatocellular expression of glutamine synthetase: an indicator of morphogen actions as master regulators of zonation in adult liver. Prog Histochem Cytochem 41, 201-266 (2007).

24. A. Dhawan, S. C. Strom, E. Sokal, I. J. Fox, Human hepatocyte transplantation. Methods Mol Biol 640, 525-534 (2010).

25. N. Kobayashi, M. Ito, J. Nakamura, J. Cai, C. Gao, J. M. Hammel, I. J. Fox, Hepatocyte transplantation in rats with decompensated cirrhosis. Hepatology 31, 851-857 (2000).

26. B. Thornburg, K. Desai, R. Hickey, L. Kulik, D. Ganger, T. Baker, M. Abecassis, R. J. Lewandowski, R. Salem, Portal Vein Recanalization and Transjugular Intrahepatic Portosystemic Shunt Creation for Chronic Portal Vein Thrombosis: Technical Considerations. Tech Vasc Interv Radiol 19, 52-60 (2016).

27. C. M. Habibullah, I. H. Syed, A. Qamar, Z. Taher-Uz, Human fetal hepatocyte transplantation in patients with fulminant hepatic failure. Transplantation 58, 951-952 (1994).

28. G. Senti, B. M. Prinz Vavricka, I. Erdmann, M. I. Diaz, R. Markus, S. J. McCormack, J. J. Simard, B. Wuthrich, R. Crameri, N. Graf, P. Johansen, T. M. Kundig, Intralymphatic allergen administration renders specific immunotherapy faster and safer: a randomized controlled trial. Proc Nat/ Acad Sci U S A 105, 17908-17912 (2008).

29. A. Dhawan, J. Puppi, R. D. Hughes, R. R. Mitry, Human hepatocyte transplantation: current experience and future challenges. Nat Rev Gastroenterol Hepatol 7, 288-298 (2010).

30. S. Mattapally, K. M. Pawlik, V. G. Fast, E. Zumaquero, F. E. Lund, T. D. Randall, T. M. Townes, J. Zhang, Human Leukocyte Antigen Class I and II Knockout Human Induced Pluripotent Stem CellDerived Cells: Universal Donor for Cell Therapy. J Am Heart Assoc 7, e010239 (2018).

31. T. Takebe, R. R. Zhang, H. Koike, M. Kimura, E. Yoshizawa, M. Enomura, N. Koike, K. Sekine, H. Taniguchi, Generation of a vascularized and functional human liver from an iPSC-derived organ bud transplant. Nat Protoc 9, 396-409 (2014).

32. M. Grompe, S. Strom, Mice with human livers. Gastroenterology 145, 1209-1214 (2013).

33. A. C. Hunt, Micro-anatomy of the lymph nodes of the pig. Br J Exp Pathol 49, 338-339 (1968).

34. D. E. McFarlin, R. M. Binns, Lymph node function and lymphocyte circulation in the pig. Adv Exp Med Biol 29, 87-93 (1973).

35. C. T. Nicolas, R. D. Hickey, K. L. Allen, Z. Du, R. M. Guthman, R. A. Kaiser, B. Amiot, A. Bansal, M. K. Pandey, L. Suksanpaisan, T. R. DeGrado, S. L. Nyberg, J. B. Lillegard, Hepatocyte spheroids as an alternative to single cells for transplantation after ex vivo gene therapy in mice and pig models. Surgery 164, 473-481 (2018).

36. R. D. Hickey, J. B. Lillegard, J. E. Fisher, T. J. McKenzie, S. E. Hofherr, M. J. Finegold, S. L. Nyberg, M. Grompe, Efficient production of Fah-null heterozygote pigs by chimeric adeno-associated virusmediated gene knockout and somatic cell nuclear transfer. Hepatology 54, 1351-1359 (2011).

37. R. D. Hickey, S. A. Mao, J. Glorioso, J. B. Lillegard, J. E. Fisher, B. Amiot, P. Rinaldo, C. O. Harding, R. Marler, M. J. Finegold, M. Grompe, S. L. Nyberg, Fumarylacetoacetate hydrolase deficient pigs are a novel large animal model of metabolic liver disease. Stem Cell Res 13, 144-153 (2014).

38. T. D. Sielaff, M. Y. Hu, S. Rao, K. Groehler, D. Olson, H. J. Mann, R. P. Remmel, R. A. Shatford, B. Amiot, W. S. Hu, et al., A technique for porcine hepatocyte harvest and description of differentiated metabolic functions in static culture. Transplantation 59, 1459-1463 (1995).

39. M. K. Pandey, A. Bansal, H. P. Engelbrecht, J. F. Byrne, A. B. Packard, T. R. DeGrado, Improved production and processing of (8)(9)Zr using a solution target. Nucl Med Biol 43, 97-100 (2016).

40. M. K. Pandey, H. P. Engelbrecht, J. P. Byrne, A. B. Packard, T. R. DeGrado, Production of 89Zr via the $89 Y(p, n) 89 Z r$ reaction in aqueous solution: effect of solution composition on in-target chemistry.

Nucl Med Biol 41, 309-316 (2014). 
41. A. Bansal, M. K. Pandey, Y. E. Demirhan, J. J. Nesbitt, R. J. Crespo-Diaz, A. Terzic, A. Behfar, T. R. DeGrado, Novel (89)Zr cell labeling approach for PET-based cell trafficking studies. EJNMMI Res 5, 19 (2015).

42. R. D. Hickey, S. A. Mao, B. Amiot, L. Suksanpaisan, A. Miller, R. Nace, J. Glorioso, M. K. O'Connor, K. W. Peng, Y. Ikeda, S. J. Russell, S. L. Nyberg, Noninvasive 3-dimensional imaging of liver regeneration in a mouse model of hereditary tyrosinemia type 1 using the sodium iodide symporter gene. Liver Transp/ 21, 442-453 (2015).

43. A. M. Shapiro, Islet transplantation in type 1 diabetes: ongoing challenges, refined procedures, and long-term outcome. Rev Diabet Stud 9, 385-406 (2012).

44. H. Jiang, A. Bansal, M. K. Pandey, K. W. Peng, L. Suksanpaisan, S. J. Russell, T. R. DeGrado, Synthesis of 18F-Tetrafluoroborate via Radiofluorination of Boron Trifluoride and Evaluation in a Murine C6Glioma Tumor Model. J Nucl Med 57, 1454-1459 (2016).

45. M. Martin, Cutadapt removes adapter sequences from high-throughput sequencing reads. 2011 17, 3 (2011).

46. H. Li, R. Durbin, Fast and accurate short read alignment with Burrows-Wheeler transform. Bioinformatics 25, 1754-1760 (2009). 
Acknowledgements: We thank T. Wyman and L. Filzen for large animal PET imaging support; L. Gross and L. Acosta for histology support; S. Krage and J Pederson for surgical support; L. Hillin for animal support. P. Steiner for graphical support. Funding: Funding for this study came from NIH K01 DK106056 award, Mayo CTSA grant number UL1TR000135, Regenerative Medicine Minnesota RMM 101617TR002, Children's Hospital of Minnesota Foundation, NIH R01 DK1 14282 (B.H. and E.L.), Commonwealth of Pennsylvania SAP4100073573 (M.G.F and E.L.) and Ri.MED Foundation (M.G.F.). Author contributions: CN, RH, RK, and JL conducted experiments and wrote the manuscript. SN designed experiments. EL designed experiments, interpreted results, and revised the manuscript. $\mathrm{BH}, \mathrm{MF}, \mathrm{AC}, \mathrm{KA}, \mathrm{RG}, \mathrm{CV}$, BA conducted experiments. ZD developed reagents and conducted experiments. LS, HJ, AB, MP, IG, VL, AB, DO, JK, TD, provided expertise and interpreted data. Competing interests: the authors have no conflicts of interest. Data and materials availability: The datasets generated and/or analyzed during the current study are available from the corresponding author on reasonable request. 
bioRxiv preprint doi: https://doi.org/10.1101/648493; this version posted May 29, 2019. The copyright holder for this preprint (which was not certified by peer review) is the author/funder. All rights reserved. No reuse allowed without permission.

Table 1. Summary of cell transplantations

\begin{tabular}{|c|c|c|c|c|c|c|c|c|c|}
\hline $\begin{array}{l}\mathrm{Pig} \\
\text { ID }\end{array}$ & Sex & $\begin{array}{c}\text { Weight at } \\
\text { Transplant } \\
\text { (kg) }\end{array}$ & $\begin{array}{l}\text { Live Cells } \\
\text { Harvested } \\
\left(\times 10^{6}\right)\end{array}$ & $\begin{array}{c}\text { Viability } \\
(\%)\end{array}$ & $\begin{array}{l}\text { Injection } \\
\text { Volume of } \\
\text { Saline }(\mathrm{mL})\end{array}$ & $\begin{array}{l}\text { Number of } \\
\text { Lymph Nodes } \\
\text { Injected }\end{array}$ & 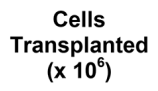 & $\begin{array}{c}\text { Cells } \\
\text { Transplanted } \\
\left(\times 10^{6} / \mathrm{kg}\right)\end{array}$ & $\begin{array}{c}\text { Days of } \\
\text { Follow-up }\end{array}$ \\
\hline 265 & Female & 20.8 & 1160 & 84 & 10 & $10-20$ & 600 & 28.8 & 239 \\
\hline 268 & Male & 19.8 & 770 & 77 & 20 & $10-20$ & 600 & 30.3 & 235 \\
\hline 270 & Male & 16.4 & 995 & 82 & 20 & $10-20$ & 600 & 36.6 & 241 \\
\hline 272 & Male & 19.0 & 975 & 74 & 20 & $10-20$ & 600 & 31.6 & 212 \\
\hline 278 & Female & 19.6 & 671 & 85 & 20 & $10-20$ & 600 & 30.6 & 239 \\
\hline
\end{tabular}



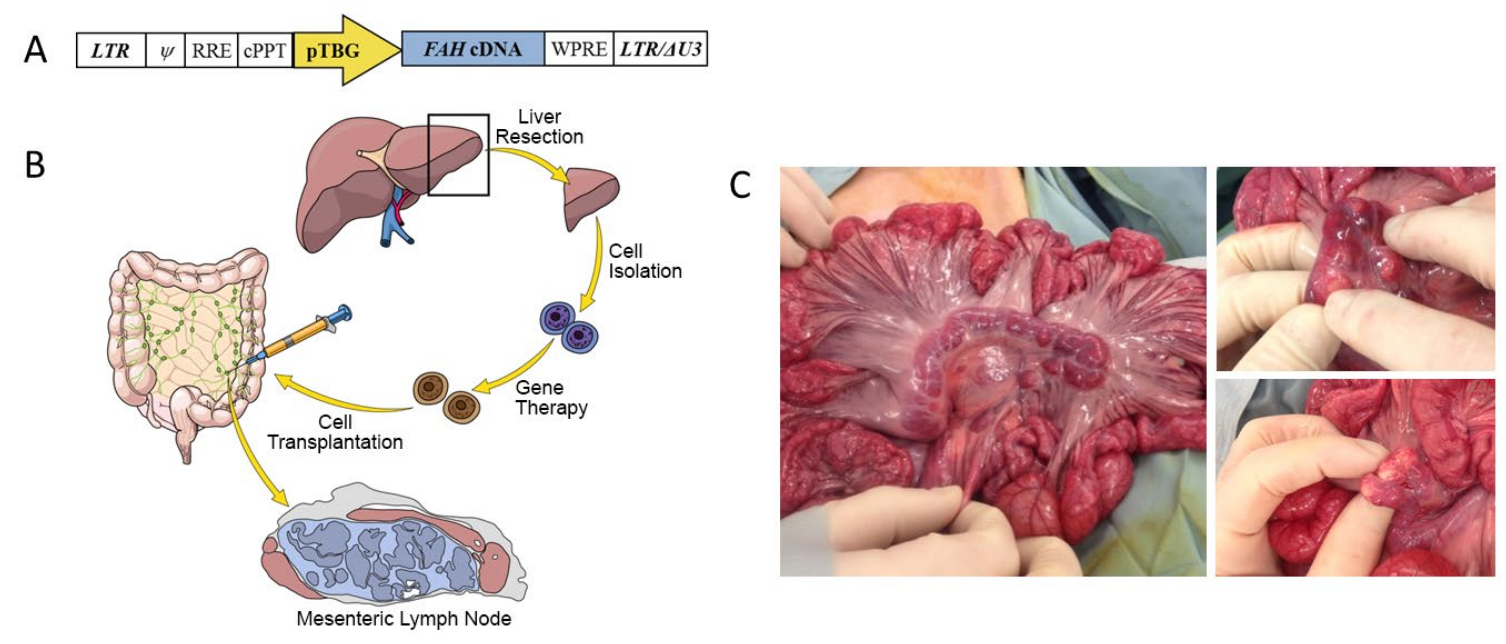

D
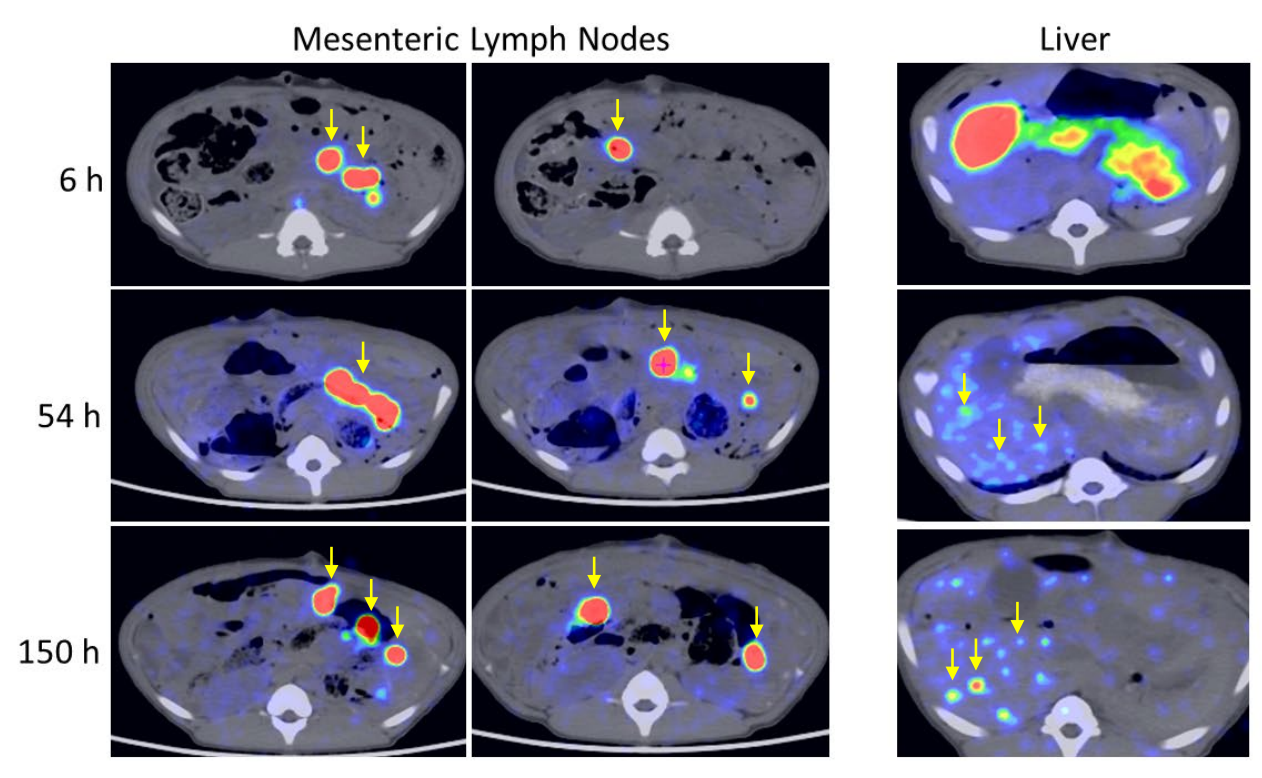

Figure 1. Schematic representation of the lentiviral vector and experimental process, and PET-CT images of 89Zr-labeled hepatocytes at 6, 54, and $150 \mathrm{~h}$ post-transplantation into pig mesenteric lymph nodes. (A) The human FAH cDNA is under control of the pig thyroxinebinding globulin (pTBG) promoter. LTR, long terminal repeat; $\Psi$, psi packaging sequence; RRE, Rev-responsive element; cPPT, central polypurine tract; LTR/ $\Delta \mathrm{U} 3$, 3' long terminal repeat with deletion in U3 region. (B) Four steps are involved in this process: 1) partial hepatectomy, 2) primary hepatocyte isolation through collagenase perfusion, 3) ex vivo gene therapy with a lentiviral vector carrying the human $F A H$ gene, and 4) transplantation of autologous hepatocytes into the mesenteric lymph nodes. (C) Transplantation of hepatocytes into mesenteric lymph nodes. Macroscopic appearance of the mesenteric lymph nodes before (left) and after (right) hepatocyte 
transplantation. (D) Representative axial images showing presence of ${ }^{89} \mathrm{Zr}$-labeled hepatocytes in the mesenteric lymph nodes within $6 \mathrm{~h}$ of transplantation in a single pig, and engraftment maintained at nearly a week post-transplantation. At the 54 and $150 \mathrm{~h}$ time-points, hepatocyte engraftment is observed within the liver as well as in the mesenteric lymph nodes. Representative foci of positive hepatocytes are indicated by yellow arrows (all panels). 
A

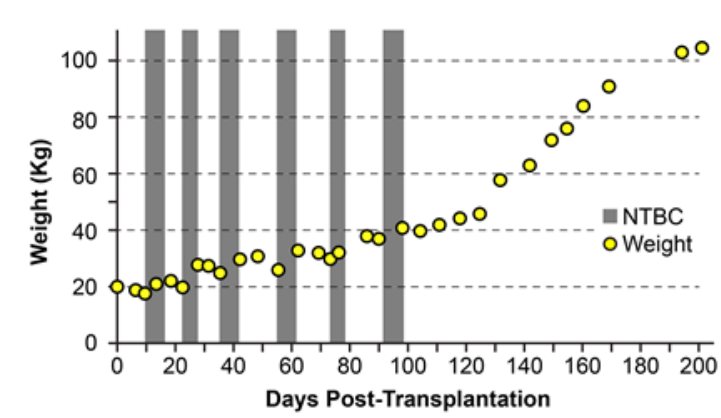

278

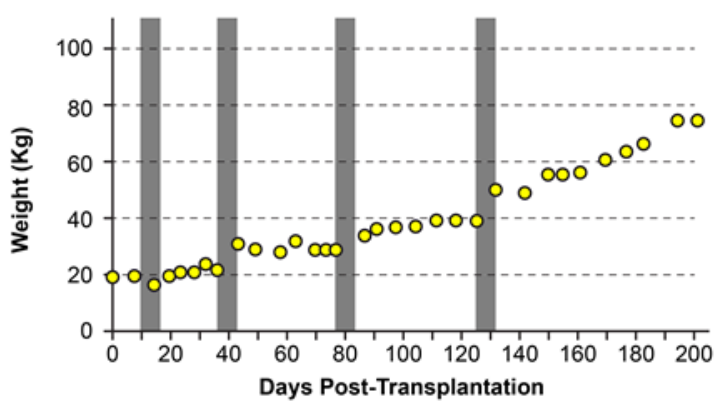

B
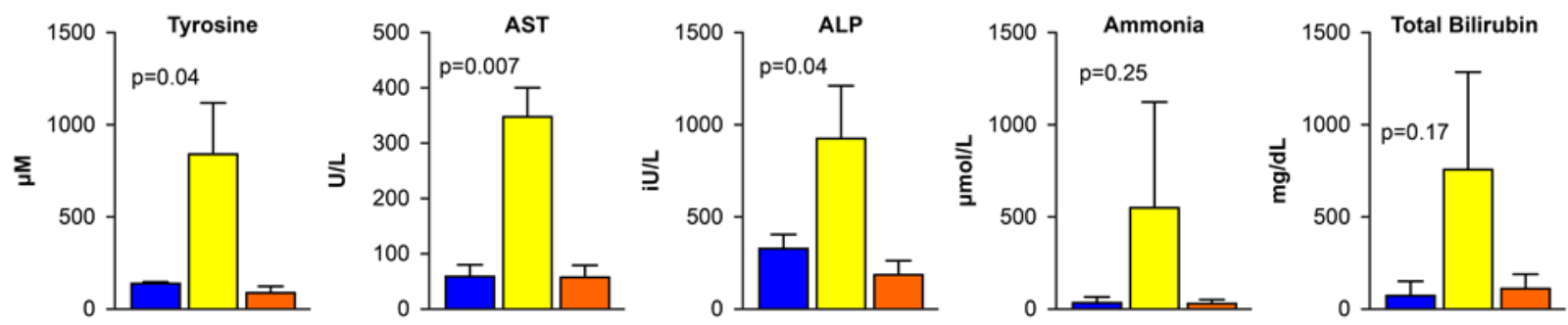

Wild-Type

$\square$ Fah $^{-\alpha} \quad \square$ Treated Fah ${ }^{-1-}$

Figure 2. Weight gain and biochemical correction of treated pigs. (A) Weight stabilization of pigs 272 and 278 demonstrating NTBC-independent growth at days 97 and 131 posttransplantation. (B) Normalization of tyrosine, aspartate aminotransferase (AST), alkaline phosphatase (ALP), ammonia, and total bilirubin levels at the time of euthanization in all animals. Mean \pm SD are shown for experimental animal cohort (treated $\mathrm{Fah}^{-/-}$animals) alongside historical wild-type and untreated $\mathrm{Fah}^{-/-}$control animals. P-values are provided for experimental animal cohort compared to untreated $\mathrm{Fah}^{-/-}$controls. 
A


B

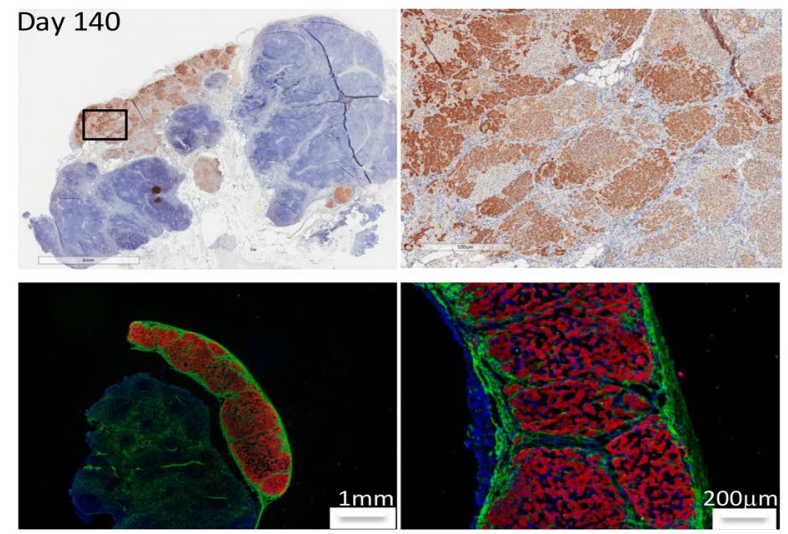

CK18/ER-TR7/Hoechst
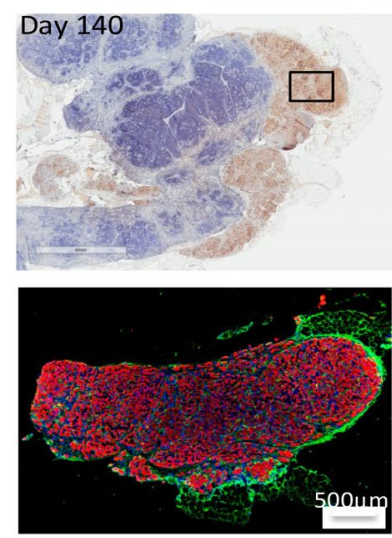

CK18/ER-TR7/Hoechst

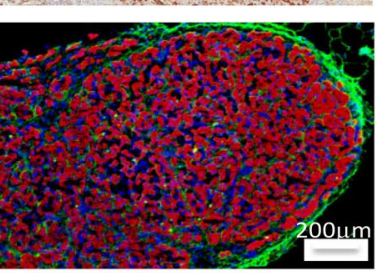

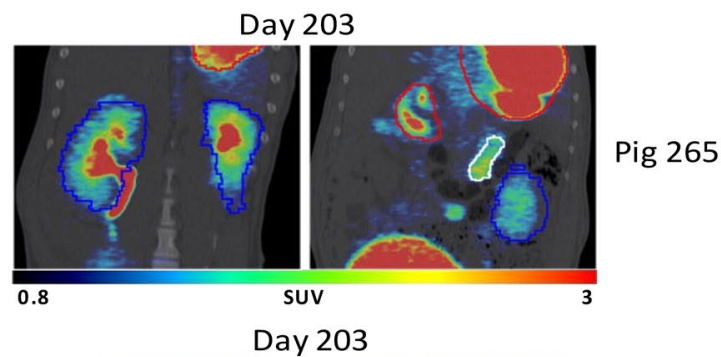

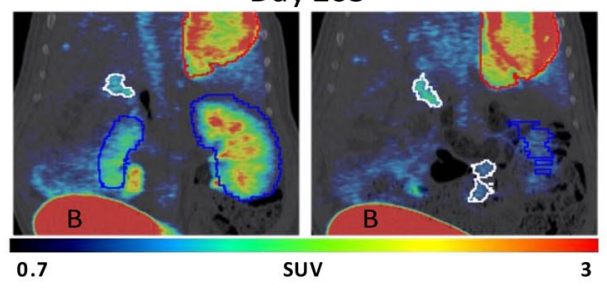

Pig 268
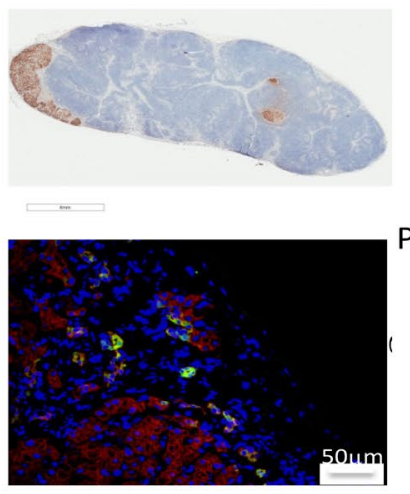

CK7/CK18/Hoechst

Pig 265

Day 235
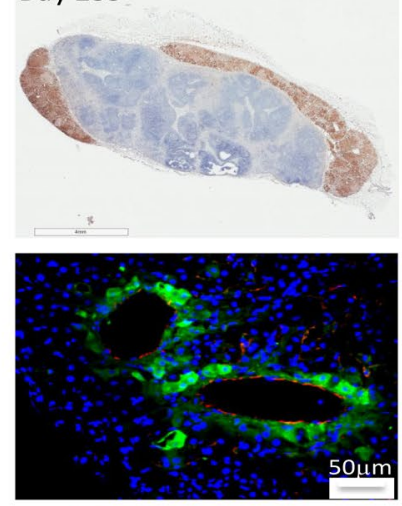

GS/CD31/Hoechst

Pig 268

Figure 3. Long-term presence of ectopic liver tissue in mesenteric lymph nodes. (A) PET-CT images of NIS-labeled hepatocytes at 104, 177, and 203 days post-transplantation into mesenteric lymph nodes. Representative coronal images showing persistent engraftment of NIS-labeled hepatocytes in the mesenteric lymph nodes in pigs 265 and 268. Mesenteric lymph nodes are 
outlined in white; stomach is outlined in red and kidney in blue; bladder is labeled as "B". (B) Histological confirmation of ectopic hepatocyte presence in mesenteric lymph nodes at 140 and 235-239 days post-transplantation. Pig 265: IHC showing presence of FAH-positive hepatocytes at days 140 (top left, middle) and 239 (top right) post-transplantation. Immunofluorescence at day 239 showing cytokeratin-18 (CK18, red) positive hepatocytes and reticular fibroblasts (ER-TR7, green) on Hoechst background (bottom left, middle), and cytokeratin-7 (CK7, green) positive bile duct cells (bottom right). Pig 268: IHC showing presence of FAH-positive hepatocytes at days 140 (top left, middle) and 235 (top right) post-transplantation. Immunofluorescence at day 235 showing cytokeratin-18 (CK18, red) positive hepatocytes and reticular fibroblasts (ER-TR7, green) on Hoechst background (bottom left, middle), and glutamine synthetase (GS, green) positive hepatocytes around CD31-positive endothelial cells (red) forming possible central vein (bottom right). 


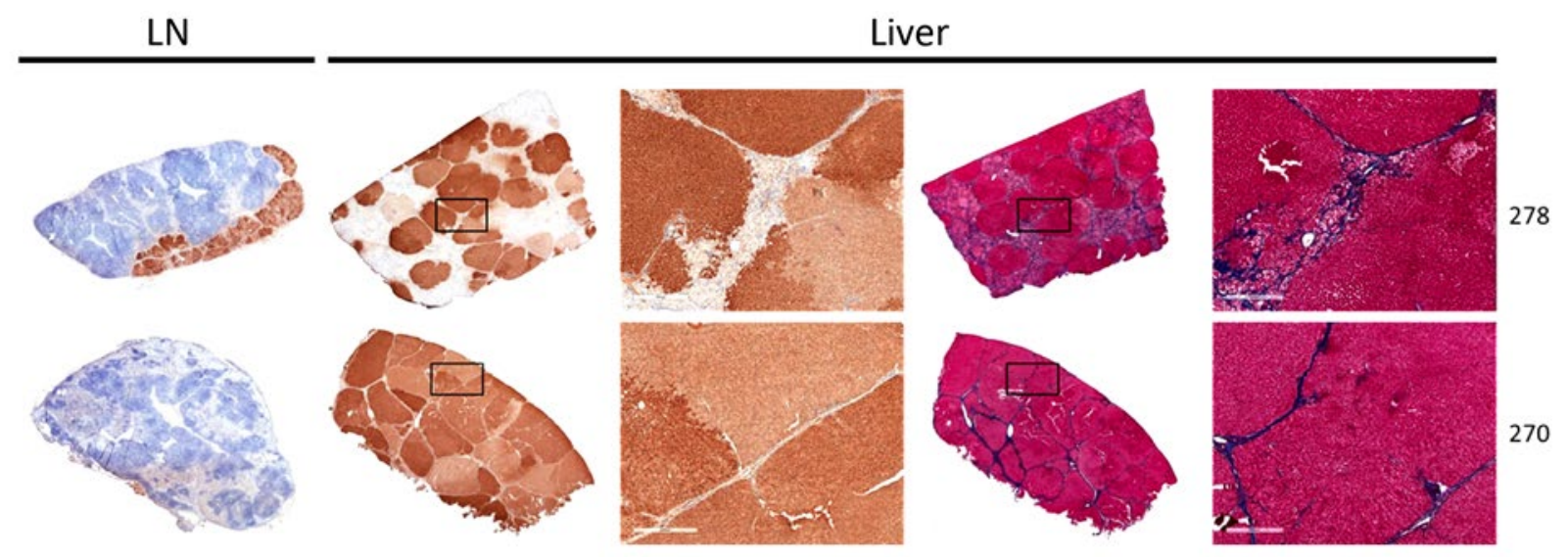

Figure 4. Liver repopulation with FAH-positive hepatocytes. Pig 278: IHC showing presence of FAH-positive hepatocytes in mesenteric lymph node (left) and liver (center) at day 239 posttransplantation; Trichrome (right) showing hepatocellular damage and fibrosis in FAH-negative areas of the liver at day 239 post-transplantation. Pig 270: IHC showing minimal presence of FAHpositive hepatocytes in mesenteric lymph node (left) and complete liver repopulation with FAHpositive cells (center) at day 241 post-transplantation; Trichrome (right) showing resolution of fibrosis with minimal residual scarring in FAH-repopulated livers at day 241 post-transplantation. 

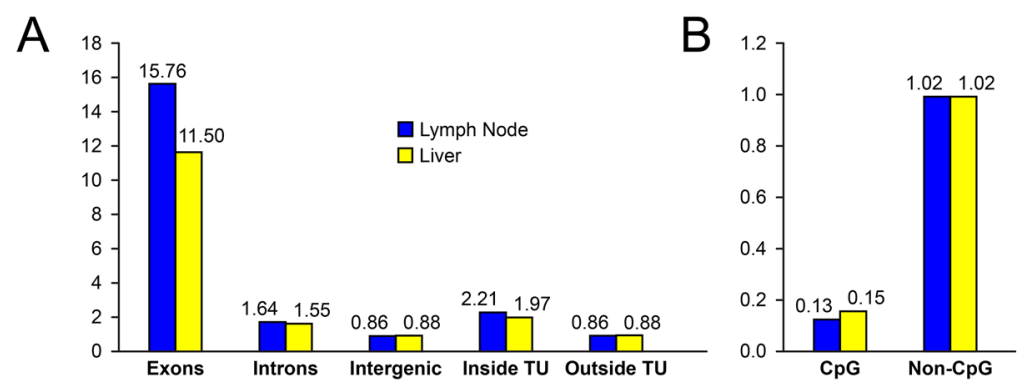

C

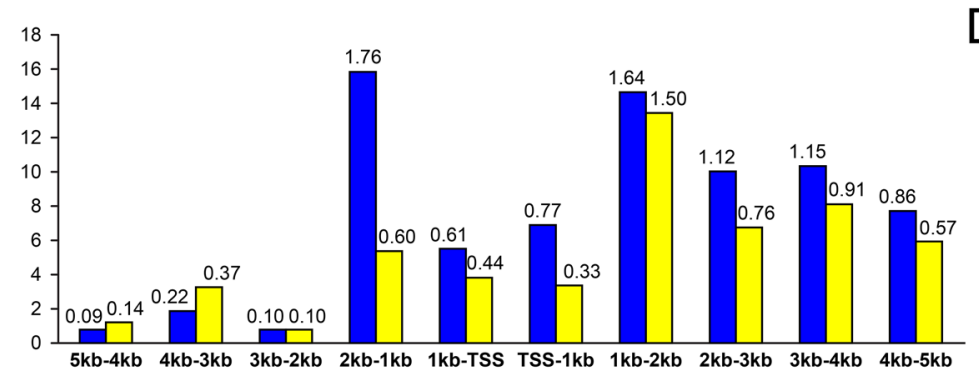

E

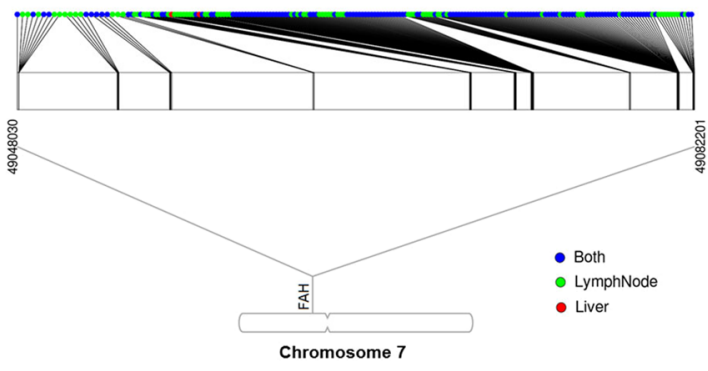

F
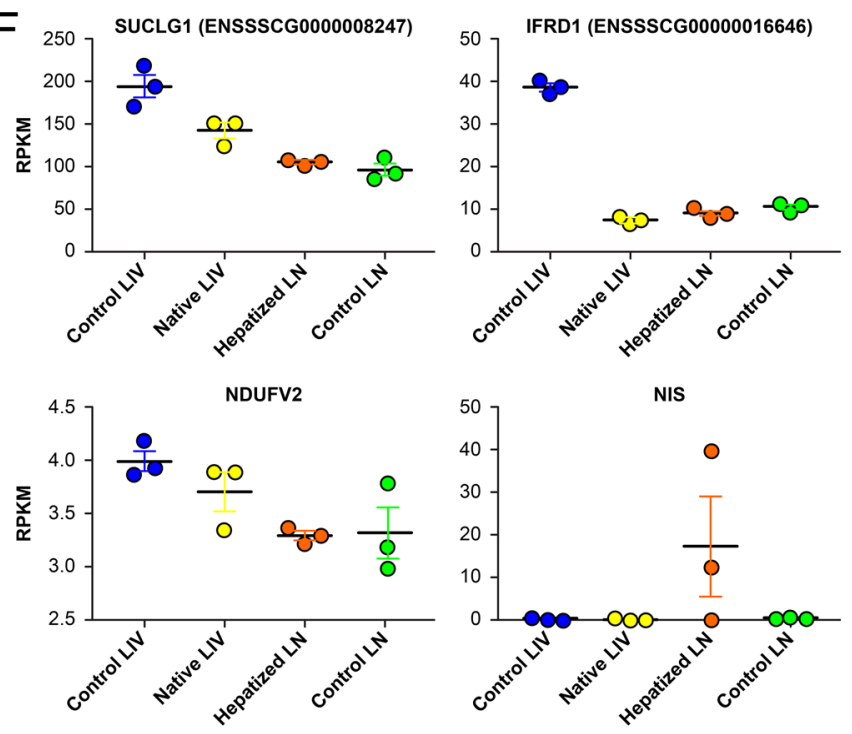

Figure 5. Lentiviral integration profile in lymph node vs. liver hepatocytes. (A) Relative integration frequency in exons, introns, intergenic regions, inside transcription units (TU), and 
outside transcriptions units for transplanted hepatocytes that remained in lymph nodes and transplanted hepatocytes that migrated to the liver. (B) Relative integration frequency in $\mathrm{CpG}$ rich promoter regions vs. other areas of the genome for transplanted hepatocytes that remained in lymph nodes and transplanted hepatocytes that migrated to the liver. (C) Relative integration frequency based on distance upstream and downstream of transcription start sites for transplanted hepatocytes that remained in lymph nodes and transplanted hepatocytes that migrated to the liver. (D) Relative integration frequency in tumor-associated genes vs. other areas of the genome for transplanted hepatocytes that remained in lymph nodes and transplanted hepatocytes that migrated to the liver. (E) Lenti integration into the fah locus, $99 \%$ of the clones found in the liver were clones of cells that first engrafted in the lymph nodes and then migrated to the liver. (F) Scatter dot plot graphs showing RPKM values for liver-enriched lenti-disrupted genes and the reporter gene (NIS) in control liver, native liver, hepatized lymph node, and control lymph node. Data are mean \pm SEM. 
bioRxiv preprint doi: https://doi.org/10.1101/648493; this version posted May 29, 2019. The copyright holder for this preprint (which was not certified by peer review) is the author/funder. All rights reserved. No reuse allowed without permission.

Table 2. Genes with most frequent LV-Fah integration. Enrichment was calculated as the percent of reads in the liver population divided by the percent of reads in the lymph node population of hepatocytes for specific gene.

\begin{tabular}{|c|c|c|c|c|c|c|c|c|c|c|}
\hline \multicolumn{5}{|c|}{ Lymph node Hepatocytes } & \multicolumn{6}{|c|}{ Liver Hepatocytes } \\
\hline $\begin{array}{c}\text { Top } \\
\text { Genes }\end{array}$ & $\begin{array}{l}\text { Number of } \\
\text { Integration } \\
\text { Sites }\end{array}$ & $\begin{array}{c}\text { Percent of } \\
\text { Integration } \\
\text { Sites }\end{array}$ & $\begin{array}{l}\text { Number } \\
\text { of Reads }\end{array}$ & $\begin{array}{l}\text { Percent } \\
\text { of Reads }\end{array}$ & $\begin{array}{c}\text { Top } \\
\text { Genes }\end{array}$ & $\begin{array}{c}\text { Number of } \\
\text { Integration } \\
\text { Sites }\end{array}$ & $\begin{array}{c}\text { Percent of } \\
\text { Integration } \\
\text { Sites }\end{array}$ & $\begin{array}{c}\text { Number of } \\
\text { Reads }\end{array}$ & $\begin{array}{l}\text { Percent } \\
\text { of Reads }\end{array}$ & Enrichment \\
\hline GLRX2 & 26 & 0.40 & 338,309 & 25.57 & MIR9799 & 18 & 0.41 & $5,479,826$ & 56.29 & 5.00 \\
\hline MIR9799 & 14 & 0.21 & 148,852 & 11.24 & NDUFV2 & 53 & 1.20 & $1,669,810$ & 17.15 & 5.76 \\
\hline LNPK & 53 & 0.81 & 146,239 & 11.05 & IFRD1 & 45 & 1.02 & 952,259 & 9.79 & 5.81 \\
\hline PDCD10 & 81 & 1.24 & 129,982 & 9.82 & SUCLG1 & 24 & 0.54 & 707,921 & 7.27 & 5.06 \\
\hline BEX1 & 51 & 0.78 & 41,620 & 3.15 & CENPP & 77 & 1.75 & 328,807 & 3.38 & 78.69 \\
\hline NDUFV2 & 25 & 0.38 & 39,429 & 2.98 & CPX2 & 36 & 0.82 & 78,896 & 0.81 & 5.63 \\
\hline ID01 & 5 & 0.08 & 30,360 & 2.29 & CTDSP2 & 45 & 1.02 & 41,364 & 0.42 & 13.71 \\
\hline FAH & 206 & 3.15 & 28,545 & 2.16 & RAB28 & 19 & 0.43 & 23,253 & 0.24 & 6.03 \\
\hline IFRD1 & 31 & 0.47 & 22,270 & 1.68 & QKI & 7 & 0.16 & 16,777 & 0.17 & 5.44 \\
\hline BMP5 & 35 & 0.54 & 19,409 & 1.47 & GSTO1 & 21 & 0.48 & 14,074 & 0.14 & 7.22 \\
\hline MTRF1 & 8 & 0.99 & 19,077 & 1.44 & ENY2 & 30 & 0.68 & 12,470 & 0.13 & 3.97 \\
\hline SUCLG1 & 53 & 0.15 & 19,033 & 1.44 & PRMT6 & 26 & 0.59 & 12,390 & 0.13 & 0.25 \\
\hline ADAMTS 1 & 5 & 0.26 & 17,754 & 1.34 & C1QTNF3 & 49 & 1.11 & 11,544 & 0.12 & 1.44 \\
\hline HSD17B3 & 32 & 0.54 & 16,484 & 1.25 & RGS5 & 13 & 0.30 & 11,377 & 0.12 & 2.02 \\
\hline SLC9A2 & 15 & 0.12 & 14,970 & 1.13 & MTRF1 & 36 & 0.82 & 10,972 & 0.11 & 0.08 \\
\hline ACVR2A & 11 & 0.81 & 13,296 & 1.00 & SNCAIP & 5 & 0.11 & 10,252 & 0.11 & 0.81 \\
\hline KCNJ2 & 21 & 0.08 & 12,294 & 0.93 & CTSV & 5 & 0.11 & 9,865 & 0.10 & 5.34 \\
\hline CLDN22 & 8 & 0.49 & 12,073 & 0.91 & FAH & 135 & 3.06 & 9,650 & 0.10 & 0.05 \\
\hline
\end{tabular}



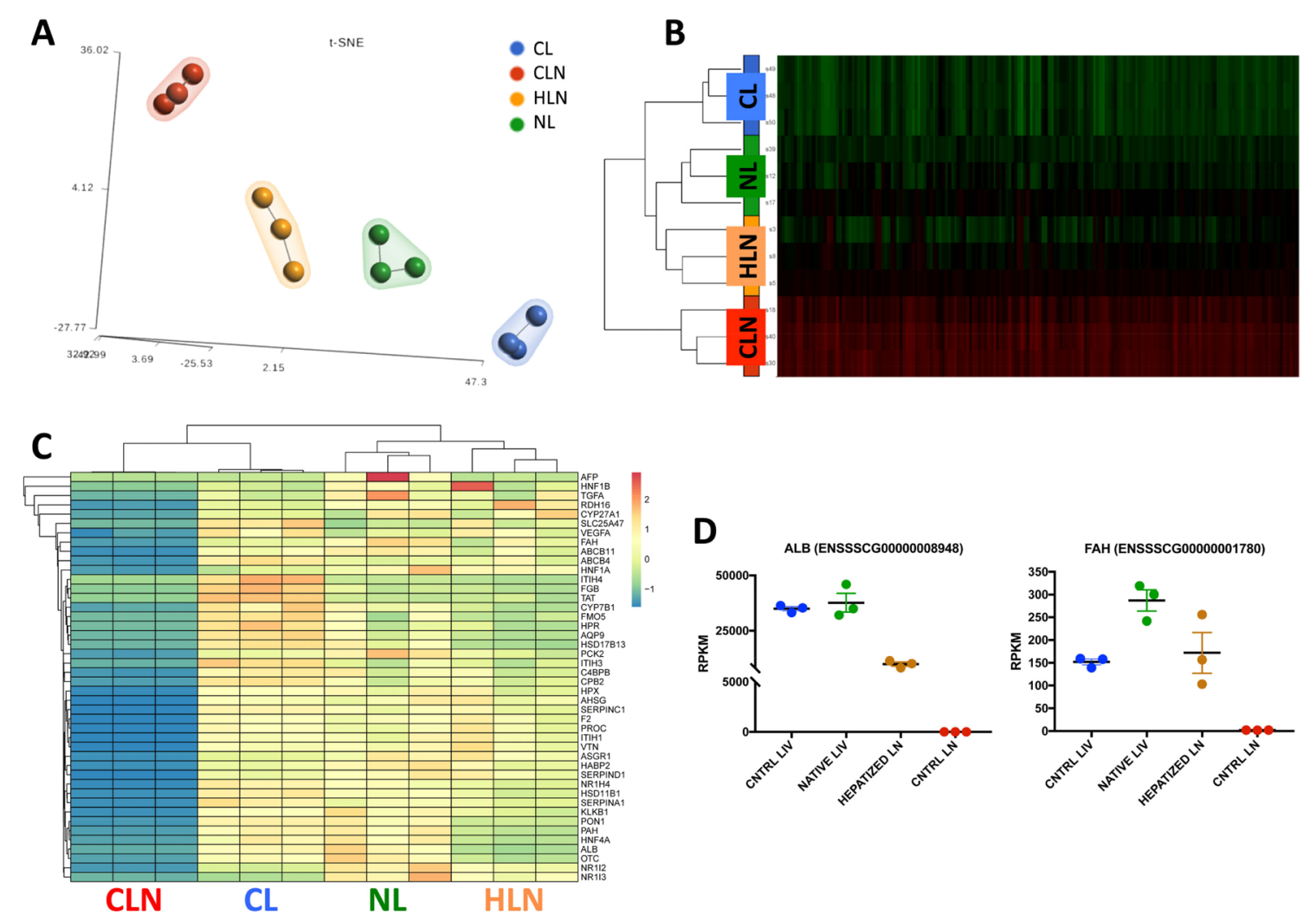

Figure 6. Transcriptional identity of hepatized lymph nodes. (A) t-Distributed stochastic neighbor embedding (t-SNE) 2D plot of RNA-seq data color-coded by tissue sample showing evidence of hepatized lymph node's (HLN) tendency to cluster with liver samples. CL, control liver; CLN, control lymph node; NL, native liver. (B) Heat map showing the normalized RNA-Seq expression levels (RPKM) of differentially expressed genes $(\log 2|\mathrm{FC}| \geq 2, \mathrm{FDR} \geq 0.05, \mathrm{n}=3)$ across the four conditions. Red and green color intensity indicate gene upregulation and downregulation, respectively, RPKM; Reads Per Kilobase Million. (C) Heat map showing the normalized RNA-Seq expression levels (RPKM) of liver-specific differentially expressed genes $(\log 2|\mathrm{FC}| \geq 2$, FDR $\geq 0.05, n=3$ ) across the four conditions. Spectral colors were used with blue indicating low expression values, yellow indicating intermediately expressed genes, and red representing highly expressed genes. (D) RPKM values for albumin (ALB) and Fah in control liver, native liver, hepatized lymph node, and control lymph node. Data are mean $\pm \mathrm{SEM}$. 


\section{Supplementary Materials}
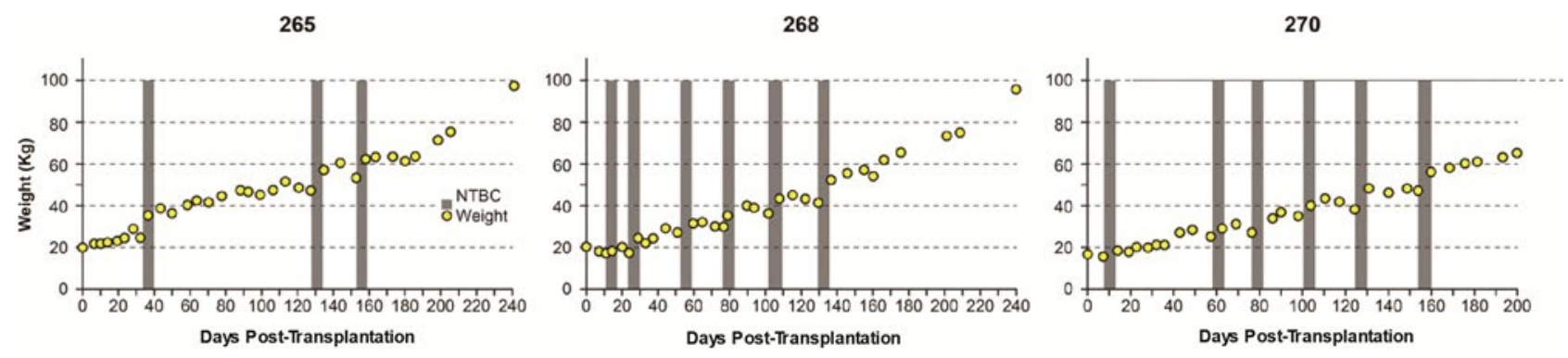

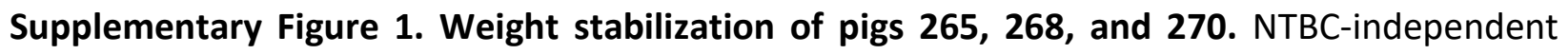

weight gain was achieved at days 158,133 , and 163 , respectively, after three to six cycles on the drug. 


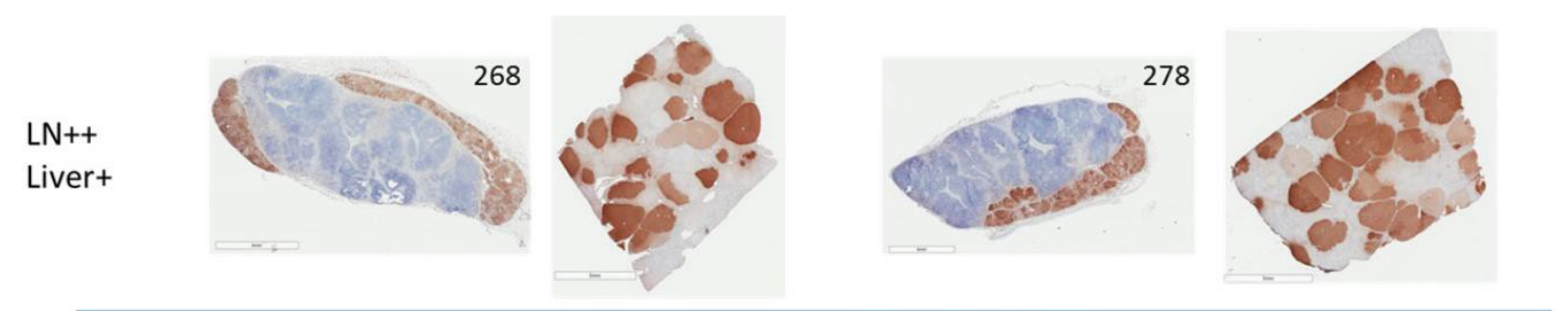

LN+

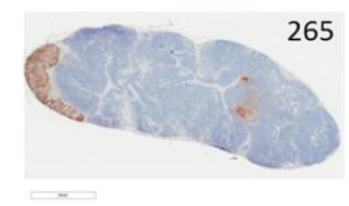

265

Liver++

270
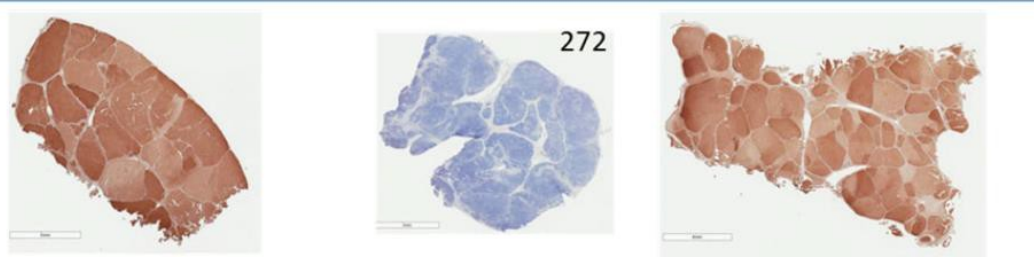

Supplementary Figure 2. Correlation between presence of FAH-positive hepatocytes in lymph

node and liver. Animals with partial liver repopulation with FAH-positive hepatocytes (pigs 268 and 278) still had robust ectopic liver tissue presence in lymph nodes at the time of euthanasia. Animals with complete liver repopulation with FAH-positive hepatocytes (pigs 270 and 272) had little to no presence of ectopic liver tissue in lymph nodes at the time of euthanasia. 
Supplementary Table 1. Next generation sequencing mapping statistics

\begin{tabular}{|c|c|c|c|c|}
\hline Sample & Total Reads & Mapped Reads (Total) & $\begin{array}{c}\text { Integration Points } \\
\text { at 1X }\end{array}$ & $\begin{array}{c}\text { Integration Points } \\
\text { at 5X }\end{array}$ \\
\hline Lymph node & 115.748 .080 & $5,465,009(7.34 \%)$ & 40.239 & 6.335 \\
\hline Liver & 116.490 .845 & $21,126,444(22.29 \%)$ & 27.987 & 4.270 \\
\hline
\end{tabular}



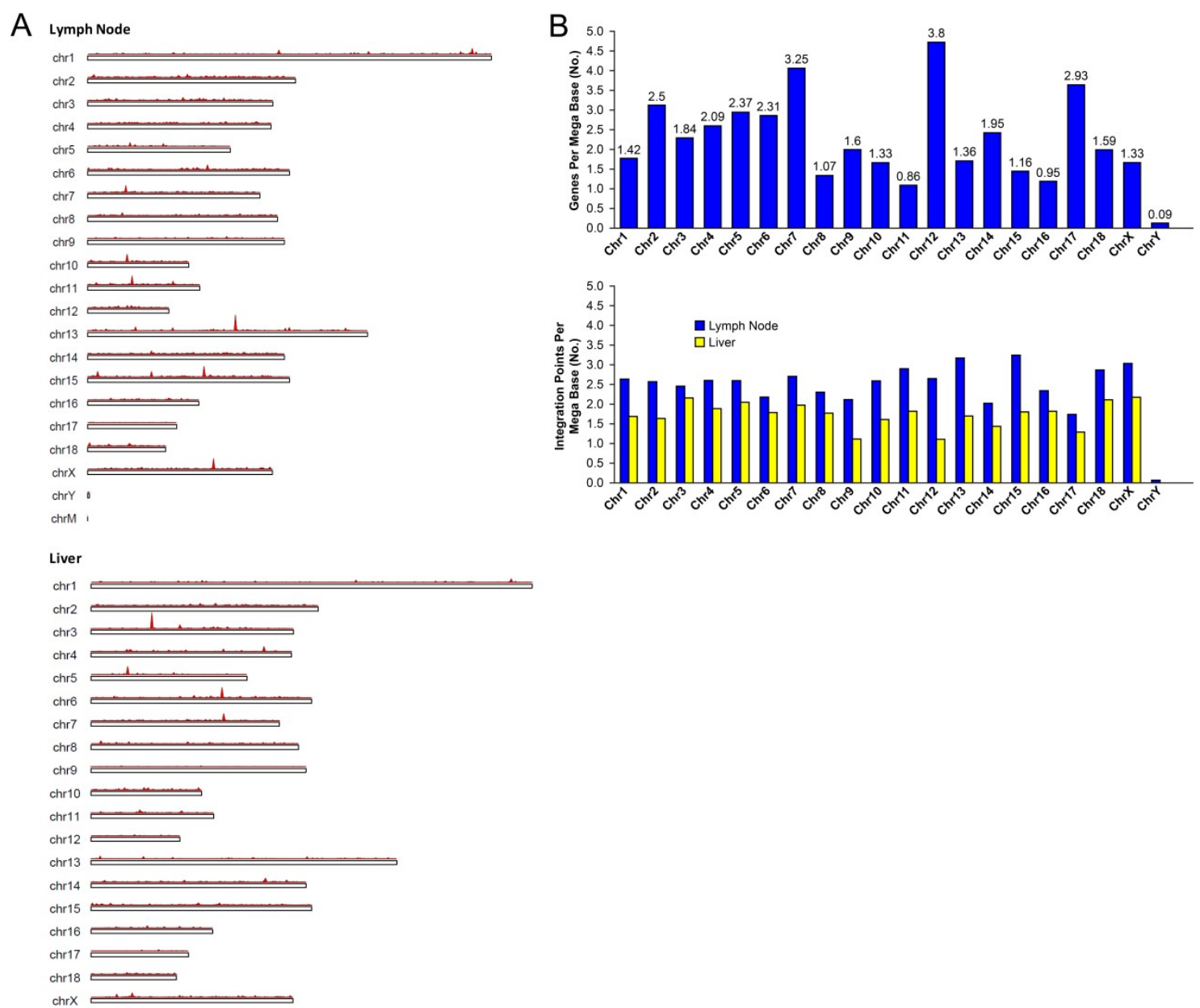

\section{Supplementary Figure 3. Lentiviral integration profile in lymph node vs. liver hepatocytes.}

(A) Chromosomal integration map for transplanted hepatocytes that remained in lymph nodes

(top) and transplanted hepatocytes that migrated to the liver (bottom). Red vertical bars represent integration points. (B) Relative gene density per chromosome (top) compared to relative integration density per chromosome (bottom). 
bioRxiv preprint doi: https://doi.org/10.1101/648493; this version posted May 29, 2019. The copyright holder for this preprint (which was not certified by peer review) is the author/funder. All rights reserved. No reuse allowed without permission.

\section{Supplementary Table 2. Liver-specific genes included in RNA sequencing analysis}

\begin{tabular}{|c|c|c|}
\hline \multicolumn{3}{|r|}{ LiGEP } \\
\hline ENSSSCG00000011799 & AHSG & Alpha 2-HS glycoprotein \\
\hline ENSSSCG00000008948 & ALB & Albumin \\
\hline ENSSSCG00000004597 & AQP9 & Aquaporin 9 \\
\hline ENSSSCG00000015661 & C4BPB & C4b-binding protein beta chain \\
\hline ENSSSCG00000009413 & CPB2 & Carboxypeptidase B2 \\
\hline ENSSSCG00000013252 & F2 & Coagulation factor II (thrombin) \\
\hline ENSSSCG00000008997 & FGB & Fibrinogen beta chain \\
\hline ENSSSCG00000006702 & FMO5 & Flavin containing monooxygenase 5 \\
\hline ENSSSCG00000010639 & HABP2 & Hyaluronan binding protein 2 \\
\hline ENSSSCG00000002749 & HPR & Haptoglobin \\
\hline ENSSSCG00000014626 & HPX & Hemopexin \\
\hline ENSSSCG00000015616 & HSD11B1 & Hydroxysteroid (11-beta) dehydrogenase 1 \\
\hline ENSSSCG00000009225 & HDD17B13 & Hydroxysteroid (17-beta) dehydrogenase 13 \\
\hline ENSSSCG00000011450 & ITIH1 & Inter-alpha-trypsin inhibitor heavy chain $\mathrm{H} 1$ \\
\hline ENSSSCG00000011451 & ITIH3 & Inter-alpha-trypsin inhibitor heavy chain $\mathrm{H} 3$ \\
\hline ENSSSCG00000011453 & ITIH4 & Inter-alpha-trypsin inhibitor heavy chain $\mathrm{H} 4$ \\
\hline ENSSSCG00000015799 & KLKB1 & Plasma kallikrein \\
\hline ENSSSCG00000015332 & PON1 & Paraoxonase 1 \\
\hline ENSSSCG00000023693 & PROC & Vitamin K-dependent protein C \\
\hline ENSSSCG00000000419 & RDH16 & Retinol dehydrogenase 16 \\
\hline ENSSSCG00000015493 & SERPINC1 & Antithrombin-III \\
\hline ENSSSCG00000010093 & SERPIND1 & Heparin cofactor 2 \\
\hline ENSSSCG00000002515 & SLC25A47 & Solute carrier family 25 member 47 \\
\hline ENSSSCG00000027801 & VTN & Vitronectin \\
\hline \multicolumn{3}{|c|}{ Additional Liver-Specific Genes } \\
\hline ENSSSCG00000016199 & CYP27A1 & Cytochrome P450 family 27 subfamily A member 1 \\
\hline ENSSSCG00000022092 & CYP7B1 & Cytochrome P450 family 7 subfamily B member 1 \\
\hline ENSSSCG00000001780 & FAH & Fumarylacetoacetate hydrolase \\
\hline ENSSSCG00000012236 & OTC & Ornithine transcarbamylase \\
\hline ENSSSCG00000000856 & $\mathrm{PAH}$ & Phenylalanine hydroxylase \\
\hline ENSSSCG00000002009 & PCK2 & Phosphoenolpyruvate carboxykinase 2 \\
\hline ENSSSCG00000002476 & SERPINA1 & Alpha-1-antitrypsin \\
\hline ENSSSCG00000002736 & TAT & Tyrosine aminotransferase \\
\hline ENSSSCG00000008949 & AFP & Alpha-fetoprotein \\
\hline ENSSSCG00000024520 & ABCB4 & $\begin{array}{l}\text { Adenosine triphosphate-binding cassette, } \\
\text { subfamily B, member } 4\end{array}$ \\
\hline ENSSSCG00000015926 & ABCB11 & Adenosine triphosphate-binding cassette B11 \\
\hline ENSSSCG00000009919 & HNF1A & Hepatocyte nuclear factor 1 homeobox $\mathrm{A}$ \\
\hline ENSSSCG00000022417 & HNF1B & Hepatocyte nuclear factor-1-beta \\
\hline ENSSSCG00000007371 & HNF4A & Hepatocyte nuclear factor 4 alpha \\
\hline ENSSSCG00000000875 & NR1H4 & Nuclear receptor subfamily 1 group $\mathrm{H}$ member 4 \\
\hline ENSSSCG00000011890 & NR112 & Nuclear receptor subfamily 1 group I member 2 \\
\hline ENSSSCG00000006353 & NR113 & Nuclear receptor subfamily 1 group I member 3 \\
\hline ENSSSCG00000024926 & ASGR1 & Asialoglycoprotein receptor 1 \\
\hline ENSSSCG00000008326 & TGFA & Transforming growth factor alpha \\
\hline ENSSSCG00000001695 & VEGFA & Vascular endothelial growth factor $A$ \\
\hline
\end{tabular}




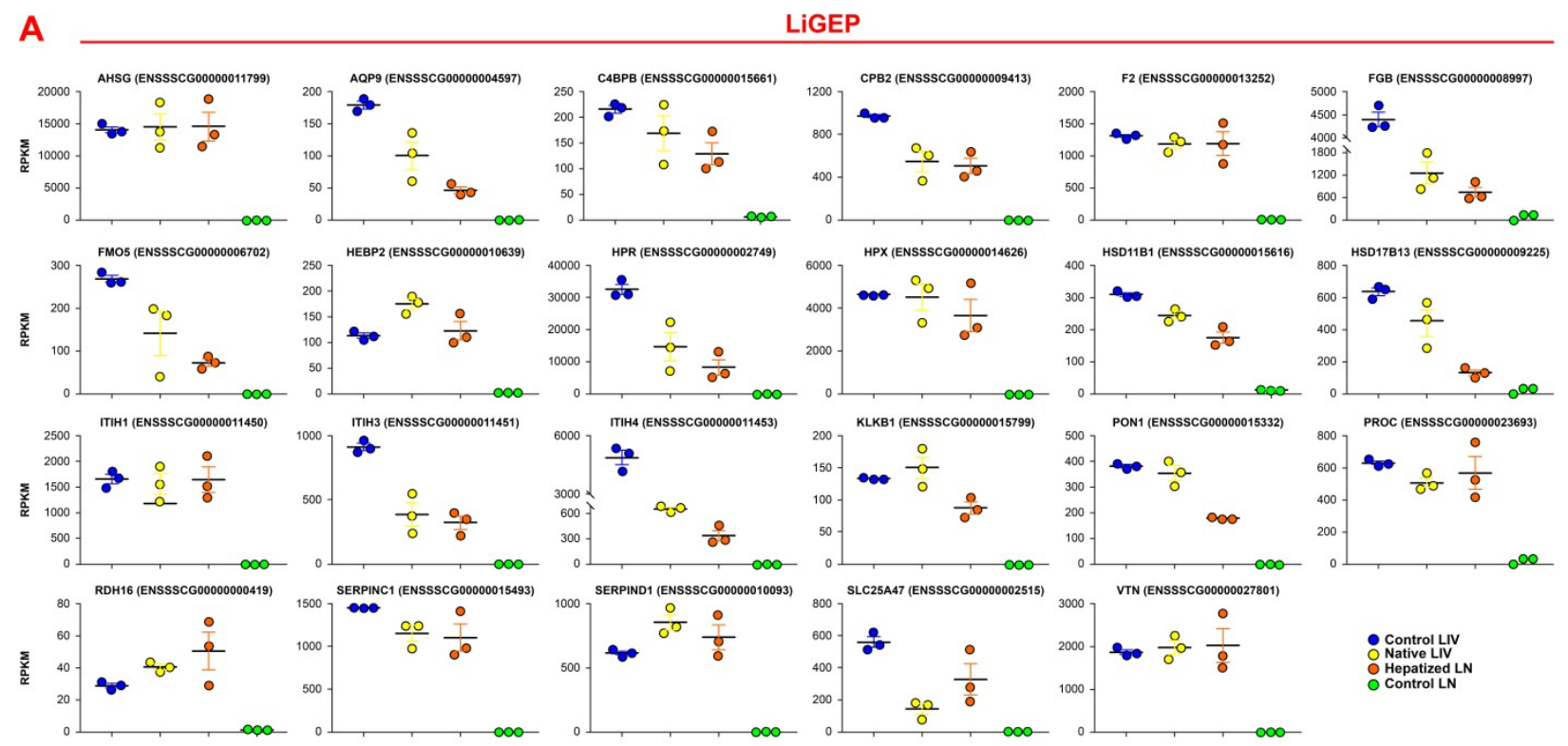

B Cytochrome P450 Genes
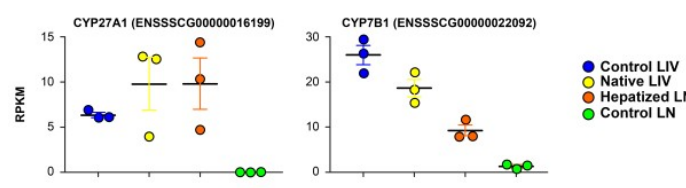
O Hepatized LN
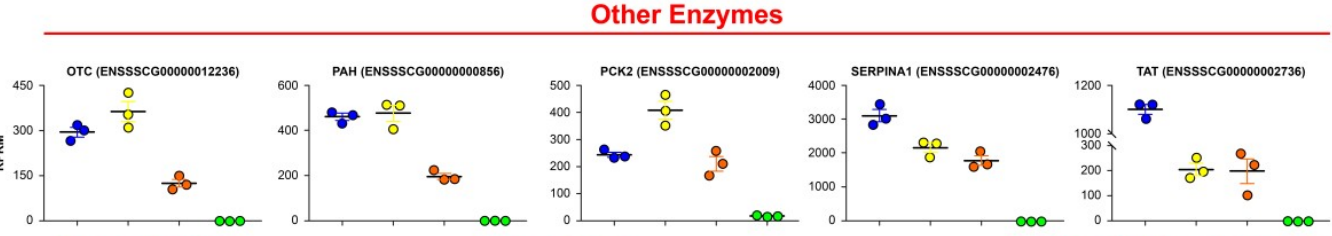

Transporters

Surface Molecules and Cytokines
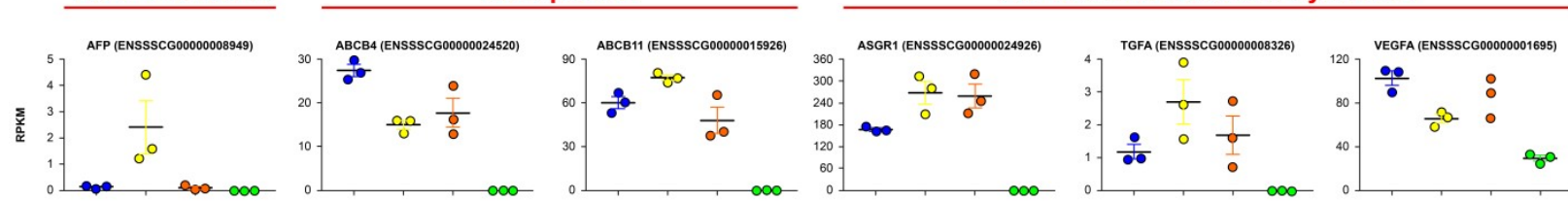

Transcription Factors


Supplementary Figure 4. Further RNA sequencing analysis. Scatter dot plot graphs showing RPKM values for LiGEP (liver-specific gene expression panel) (A) or additional liver-specific genes (B) in control liver, native liver, hepatized lymph node, and control lymph node. Data are mean \pm SEM. 
Supplementary Video 1. 3D render movie of PET-CT images of ${ }^{89} \mathrm{Zr}$-labeled hepatocytes at $6 \mathrm{~h}$ post-transplantation into mesenteric lymph nodes in pig.

Supplementary Video 2. 3D render movie of PET-CT images of NIS-labeled hepatocytes at 3 months post-transplantation into mesenteric lymph nodes in pig 265.

Supplementary Video 3. 3D render movie of PET-CT images of NIS-labeled hepatocytes at 6 months post-transplantation into mesenteric lymph nodes in pig 265.

Supplementary Video 4. 3D render movie of PET-CT images of NIS-labeled hepatocytes at 5 months post-transplantation into mesenteric lymph nodes in pig 268.

Supplementary Video 5. 3D render movie of PET-CT images of NIS-labeled hepatocytes at 6 months post-transplantation into mesenteric lymph nodes in pig 268. 